\title{
Rezensionen
}

\section{... like a mission is possible ...}

Evolution der Informationsinfrastruktur. Kooperation zwischen Bibliothek und Wissenschaft. Erschienen im Rahmen des zehnjährigen Jubiläums der Abteilung Forschung und Entwicklung der SUB Göttingen. Hrsg. von Heike Neuroth, Norbert Lossau, Andrea Rapp. Glückstadt: Verlag Werner Hülsbusch, 2013. 368 S. mit Abb.

Besprochen von Prof. Dr. Andreas Degkwitz: Direktor der Universitätsbibliothek der Humboldt-Universität zu Berlin, Unter den Linden 6, D-10099 Berlin, E-Mail: andreas.degkwitz@ub.hu-berlin.de

DOI 10.1515/bfp-2015-0034

Wie kaum ein zweites Buch beginnt diese Jubiläumsschrift zum 10-jährigen Bestehen der Forschungs- und Entwicklungsabteilung der SUB Göttingen (F\&E-Abteilung) mit seinem Cover. Dort wird der Kosmos der Digital Humanities oder E-Humanities kartiert, den sich die Akteure dieser Abteilung in den vergangenen Jahren erschlossen und in Teilen sogar erobert haben. „Archivgebirge“, „GRIDopulis“, „Open Linked Island“, „TEI-Terrassen“, „Semantisches Becken“, der „DATENSTROM“, die „Wüste GOOBI“, „WLAND“ zählen zu den systemischen Komponenten, die virtuelle Forschungsumgebungen konstituieren. Daneben finden sich das „gutachterliche Dreieck“, „die Metaebene“ und die „Milestones“, „Projektruinen“ und „Deadlines“, „der Mount Monitoring“ und die „Audit-Klippen“, die die Weiterentwicklung der Informationsinfrastrukturen - man möchte fast sagen gleichsam als „Guardians of the Galaxy“ - prägend begleiten: Wo ist der Baum als Allegorie der Nachhaltigkeit: „My name is Groot“? Geht es hier nur um die SUB Göttingen als Motor der digitalen Transformation geisteswissenschaftlicher Informationsinfrastrukturen oder ist diese „enhanced world“ über die SUB Göttingen hinaus auch von anderen „Siedlern“ bevölkert?

Die SUB Göttingen hat auf dem Gebiet der infrastrukturellen Unterstützung für digitale Geisteswissenschaften zweifellos Pionierarbeit geleistet und den Weg durch die Höhen und Tiefen dieser Mission mit Sieben-Meilen-Stiefeln durchschritten. Die Daten der gedruckten Wissenschaftstradition $\mathrm{zu}$ verarbeiten, nachdem die analogen Materialien in digitale Inhalte konvertiert worden sind, diese Herausforderung und die sich daraus ergebenden Potenziale wurden von der F\&E-Abteilung der SUB Göttingen konsequent aufgegriffen und für zahlreiche Vorhaben genutzt. Die Ergebnisse können sich weltweit sehen lassen und reichen deutlich über den Horizont des Göttinger Kosmos hinaus. Ein Überblick dessen, was erreicht worden ist, erweist sich in jedem Fall als angebracht, zumal mit den zum Durchbruch kommenden digitalen Geisteswissenschaften die Göttinger Errungenschaften nun „in die Fläche" gehen.

„Die Zeit war reif“ für eine F\&E-Abteilung überschreibt Elmar Mittler sein Kapitel der Jubiläumsschrift, in dem er die Gründung und Anfänge der Abteilung beschreibt. Elmar Mittler macht dies als Initiator und Spiritus Rector einer Entwicklung, die er selbst in Gang gesetzt hat, indem er verstand den „Kairos“, den richtigen Zeitpunkt, dafür zu nutzen. Die mit der Gunst der Stunde dann einsetzende Weiterentwicklung der Abteilung wurde und wird von seinen Nachfolgern, Norbert Lossau und Wolfram Horstmann, erfolgreich aufgegriffen. Die F\&E-Abteilung der SUB Göttingen schreibt in der Tat eine bemerkenswerte Erfolgsgeschichte, die ohne ihre langjährige Leiterin, Heike Neuroth, sicher nicht möglich gewesen wäre - das sei an dieser Stelle, an der sich der Rezensent gerne mit der Liste derer, die dazu gratulieren, „verlinken“ möchte, ganz ausdrücklich erwähnt.

Warum lohnt es sich, dieses Buch herauszubringen, und warum sollte sich seine Lektüre empfehlen, wird doch auf den ersten Blick viel Bekanntes und häufig Genanntes ein weiteres Mal zusammengefasst und den Lesern vor Augen führt? Was ist das Thema dieses Buches, das die Evolution der Informationsinfrastruktur mit 20 Beiträgen von rund 30 Autoren beschreibt? Natürlich geht es um den Paradigmenwechsel von analogen zu digitalen Informationsinfrastrukturen, bei dem es jedoch nicht bleibt. Denn dieser Wandel geht über das Thema der Infrastrukturentwicklung und die damit einhergehende Kooperation zwischen Bibliothek und Wissenschaft weit hinaus, indem die Digitalisierung geisteswissenschaftlicher Forschung verstärkt das Selbstverständnis der Geistes- und Kulturwissenschaften betrifft. „Data Driven Research“ ist das Stichwort, das diese Entwicklung charakterisiert und die Zukunft geisteswissenschaftlicher Forschung und die $\mathrm{Zu}-$ kunft der Bibliotheken prägt. Was geschieht, wenn neben die traditionelle Lektüre die maschinelle Auswertung von Texten tritt, und bisher eingesetzte Verfahren der Deutung und des Verständnisses von Texten durch Analysewerkzeuge und Referenzierungen ergänzt oder sogar ersetzt werden? Haben hermeneutische Methoden der geisteswissenschaftlichen Tradition noch Bestand oder werden diese 
zunehmend durch empirische Verfahren ersetzt, wie sie für die Naturwissenschaften kennzeichnend sind? Kommt es nun zur De-Mystifizierung und damit zugleich zur Objektivierung der Geistes- und Kulturwissenschaften, die sie „endlich“ in die unmittelbare Nachbarschaft der „Hard Sciences" rückt? Welche Qualitäten haben Offenheit und Vernetzung IT-gestützter, virtueller Arbeits- und Forschungsumgebungen im Vergleich mit herkömmlicher Kollaboration und Zusammenarbeit?

Dieses Themenspektrum ist als Leitfrage zugleich das Leitmotiv des Bandes, das zunächst in der Einführung von Matthias Schulze und Ralf Stockmann erklingt, mit der ein Rückblick auf die Entwicklung von „Open Science und Networked Science“ vor dem Hintergrund der Weiterentwicklung von Netz- und Rechnerstrukturen und der darauf aufsetzenden Möglichkeiten der wissenschaftlichen Kollaboration und Zusammenarbeit gegeben wird. Im Bericht des Kolloquiums zum zehnjährigen Bestehen der F\&E-Abteilung greifen Tobias Blanke und Axel Horstmann erneut das Leitmotiv auf, das in den Beiträgen von Ulrich MeyerDörpinghaus, Achim Oswald, Andreas Rauber, Arnulf Quadt, Anke Holler und Tobias Blanke mit hohem Wiedererkennungswert vielschichtig moduliert. Einleitend wird von Norbert Lossau zunächst die Entwicklung der F\&EAbteilung der SUB Göttingen, die dabei maßgebliche Rolle von Elmar Mittler und der erreichte Stand der Infrastrukturentwicklung ausdrücklich gewürdigt, der eindeutig zu erkennen gebe, dass die Notwendigkeit des Auf- und Ausbaus von Informationsinfrastrukturen zu „E-Humanities“ und „E-Research“ in der Strategieentwicklung der Universität Göttingen „angekommen“ sei und damit weiter verfolgt werde. In den folgenden Impulsreferaten stehen die Themen „Informationskompetenz und Qualifizierung“ (Ulrich Meyer-Dörpinghaus) und die „Neuen Aufgaben und Rollen für Wissenschaftliche Bibliotheken" (Achim Oswald) im Mittelpunkt. Beide Beiträge thematisieren die Herausforderungen und Umbrüche, die sich für das Selbstverständnis von wissenschaftlichen Bibliotheken und des künftigen Aufgabenspektrums der Mitarbeiter ergeben, wenn im Zuge der digitalen Transformation Infrastruktur und Wissenschaft in einem unmittelbaren Austausch und einer wechselseitigen Entwicklungspartnerschaft stehen.

In dem von Uwe Schwiegelshohn moderierten Teil des Kolloquiums kommen Wissenschaftler aus den Natur- und Geisteswissenschaften zu Wort. Am Beispiel der Teilchenphysik erklärt Arnulf Quadt die auf Daten beruhende Herangehensweise des Forschungsgebiets, die als Beispiel für viele Fachgebiete zur Erforschung von Naturphänomenen charakteristisch ist und seit vielen Jahren die dafür erforderlichen Infrastrukturen nutzt. Anke Holler zeigt anhand der Germanistischen Linguistik eine beispielhafte
Entwicklung in den Geisteswissenschaften, der - ähnlich naturwissenschaftlichen Forschungsvorhaben - das Datenparadigma zugrunde liegt und die sich auf diese Weise in eine verstärkt empirische Richtung bewegt. Interessant sind in diesem Zusammenhang die Veränderungen, die sich aus ihrer Perspektive dazu beobachten lassen (S. 55): empirisch erhobene Sprachdaten anstelle von introspektiven Sprecherurteilen, sprachtechnologische Werkzeuge zum Aufbau von Datensammlungen und neue Verfahren zu deren Auswertung, Widerlegung von Annahmen oder tieferes Verständnis von Phänomenen mithilfe quantitativer Methoden, Forschungsfragen neuer Art, Beschleunigung und Vereinfachung der wissenschaftlichen Arbeit und vielfältige neue Recherchemöglichkeiten, kollaborative Arbeitsweisen und die Bildung interdisziplinärer Teams aus Fachspezialisten und Infrastrukturexperten. In dem abschließenden Beitrag führt Tobias Blanke zu den Digital Humanities aus, dass es dabei „um Veränderungen nicht nur der Geisteswissenschaften, sondern auch der Computer- und Naturwissenschaften geht" (S. 57).

In den folgenden drei Kapiteln „Forschungsprojekte“, „Lehre und Qualifizierung“, „Technologien und Dienste“ werden mit Einzelbeiträgen Themen behandelt, die die Arbeit der F\&E-Abteilung der SUB Göttingen konkret prägen. Nach dem Beitrag von Kathleen M. Smith und Fotis Jannidis zu Fragen und Herausforderungen nutzerorientierter Softwareentwicklung am Beispiel von TextGrid sowie der Vorhaben „The European Holocaust Research Infrastructure“ (EHRI) und „The Collaborative European Digital Archive Infrastructure“ (CENDARI) wird von Gabriele Radecke, Mathias Göbel und Sibylle Söring zur genetisch-kritischen und kommentierten Hybrid-Edition der Notizbücher von Theodor Fontane berichtet, die auf der Basis von TextGrid erstellt wird. Dabei wird einerseits auf den editionswissenschaftlichen Teil des Vorhabens eingegangen, dem fast ausschließlich Texte zugrunde liegen. Andererseits werden die informationswissenschaftlichen Verfahren und Werkzeuge erklärt, die zur infrastrukturellen Unterstützung für Aufbereitung und Strukturierung von Texten, für deren Transkription und Kommentierung unter Einschluss der Referenzierung von Orten, Personen, Schriftstellerkollegen, weiteren Werken Fontanes etc. sowie für Publikation und langfristige Verfügbarkeit genutzt werden und im Rahmen von TextGrid zur Verfügung stehen oder zusätzlich zu entwickeln sind.

In dem Editionsvorhaben ,Johann Friedrich Blumenbach - online“, zu dem Martina Kerzel, Mike Reich und Heiko Weber berichten, geht es um die Online-Edition der Schriften und der naturhistorischen Sammlungen Johann Friedrich Blumenbachs. Dabei handelt es sich um eine „Gesamtschau“ seines Werks, mit der in die Online-Edition 
seiner Schriften auch die vielfältigen Objekte der Blumenbach'schen Sammlung integriert und zugänglich gemacht werden sollen. Über die Aufbereitung der Volltexte hinaus sind deshalb auch die Sammlungsmaterialien entsprechend zu erschließen und zu referenzieren. Damit wird die Herausforderung aufgegriffen, „wie das Verhältnis von Idee und Theorie zu den empirischen Befunden (in seinen Kontinuitäten, Diskontinuitäten und Interdependenzen) in einer „Einheit in der Vielfalt“, also in den in der OnlineEdition publizierten und tiefenerschlossenen Texten (Deskription) in Rückgriff auf die identifizierten und tiefenerschlossenen Sammlungsobjekte (Empirie) im Gesamtwerk von Blumenberg steht“. Der damit erreichte Mehrwert der multimedial angereicherten Edition gibt ganz eindeutig die Potenziale der digitalen Aufbereitung und Verfügbarkeit zu erkennen und ist in seiner Aggregation nur auf digitaler Grundlage zu realisieren.

„Geisteswissenschaftliche Aufbereitung von Daten mit TEI“ ist der Titel des Beitrages, in dem Werner Wegstein und Wolfgang Pempe die Auszeichnung und Aufbereitung digitaler Texte bzw. „(Text-)Daten“ nach den Prinzipien der „Text Encoding Initiative“ (TEI) erklären. Dass die Herausgeber diesen Beitrag in den Band aufgenommen haben, ist besonders hervorzuheben. Für die vorzügliche Qualität des Beitrages ist den beiden Autoren ausdrücklich zu danken. Die TEI-Aufbereitung digitalisierter Texte gehört zu den Grundlagen für nahezu alles, was sich unter dem Label der Digital Humanities vereint. Denn die nach TEI erfolgende XML-Strukturierung von Daten und Texten ist der Schlüssel für deren Annotierung, Aufbereitung, Recherche, Referenzierung und Weiterverarbeitung. Die Einführung, die Wegstein und Pempe in die Texterschließung und Wortauszeichnung geben, gehört schlicht zur Pflichtlektüre und erklärt die Grundlagen und die Voraussetzungen für datenbasierte, geisteswissenschaftliche Forschungen. Als beeindruckend stellen sich dabei die Genauigkeit und die Präzision heraus, mit der die Kodierung erfolgen muss, damit Forscher von den Mehrwerten verarbeitbarer Daten- und Textpräsentationen profitieren können: So werden Texte zu Daten! Dass die Codierung der digitalen Version, die sich damit einer unmittelbaren Lektüre verschließt, gerade die ursprünglich analoge, nicht codierte Leseversion zu deren Erforschung entschlüsselt, ist Naturwissenschaftlern, die Daten zur Erklärung von Naturphänomenen sammeln und generieren, sicher vertraut, doch für Geisteswissenschaftler, für die mehrheitlich die Textrezeption im Mittelpunkt steht, wahrscheinlich eher ungewohnt. Ein außerordentlich interessantes Projekt ist das bereits erwähnte Vorhaben zum Aufbau einer „European Holocaust Research Infrastructure“ (EHRI), zu dem Tobias Blanke, Veerle Vanden Daelen,
Michael Frankl, Conny Kristel, Kepa J. Rodriguez und Reto Speck berichten. Mit diesem Vorhaben werden die Möglichkeiten der Verlinkung und Vernetzung genutzt, um hochgradig verteilte Inhalte und Institutionen gezielt zu aggregieren und in semantische Kontexte zu überführen. Wie mit digitalen Editionen, die über Texte hinaus auch dreidimensionale Artefakte und Sammlungsobjekte umfassen und aufeinander bezogen zur Verfügung stehen, bietet EHRI eine Landkarte von Inhalten einschließlich ihrer Lokalisierung und kann diese Materialien auf einzelne Orte fokussieren, wie das im Beitrag genannte Beispiel des Ghettos von Theresienstadt zeigt.

Die Darstellung der Projekte verdeutlicht einerseits die Vorkehrungen zur digitalen Aufbereitung und Verarbeitung von geisteswissenschaftlich relevanten Inhalten unterschiedlicher Formate sowie die damit verbundenen Aufwände. Andererseits lässt sich an den Ergebnissen aufzeigen, welche Herausforderungen sich auf dieser Arbeitsgrundlage deutlich besser aufgreifen und bewältigen lassen als dies mit den herkömmlichen, analog zur Verfügung stehenden Arbeitsverfahren zu realisieren ist. Die Präzision, mit der Texte auszuzeichnen und zu erschließen sind, bieten eine hervorragende Basis für umfassende Recherchen und Auswertungen, die mit maschinellen Methoden einen hohen Abdeckungs- und Vollständigkeitsgrad haben. Die Visualisierungsoptionen, mit denen Auswertungs- und Evaluationsergebnisse editiert werden können, ermöglichen nicht nur die intuitive Veranschaulichung von Sachverhalten, sondern eröffnen - zumindest in der weiteren Perspektive - interaktive Diskurse zu den visuell kommunizierten und reproduzierbaren Ergebnissen. Die Aggregationen, Ausgabeformate, die Kontextualisierung und Verlinkung, die in TextGrid mit interoperablen Bilder-, Objekt- und Textsammlungen verfügbar sind, konstituieren einen projektspezifischen Kosmos, der den kompletten Forschungszyklus einschließlich seiner Infrastruktur und die Vernetzung innerhalb seiner Forschungs- oder Projektcommunity umfasst. Mit der Bezeichnung „Kosmos“ wird hier charakterisiert, was die technisch geprägten Begriffe wie „Grid“ oder „Forschungsumgebung“ in seinem enormen Potenzial zumindest nicht unmittelbar erwarten lassen.

Der das Kapitel „Forschungsprojekte“ beschließende Beitrag „Erfahrungen aus dem Projekt,WissGrid - Grid für die Wissenschaft"“ von Harry Enke und Bernadette Fritzsch fasst Herausforderungen und Rahmenbedingungen für die Aufrechterhaltung und Weiterentwicklung dieses „Kosmos“ zusammen. In diesem Zusammenhang wird darauf hingewiesen, dass virtuelle Forschungsumgebungen oft ,auf gleicher technologischer Basis aufbauen, die konkrete Umsetzung aber sehr fachspezifisch und individuell sei“, einen vergleichsweise hohen Betreuungsauf- 
wand erforderten, ihre Nachhaltigkeit nicht allein aus den „Bordmitteln“ der Projekte oder der beteiligten Institutionen gewährleistet werden könne und dem Zugang zu Forschungsdaten eine zentrale Bedeutung zukomme. Was dabei sicher auch Erwähnung finden sollte, ist der Hinweis, dass für die Nutzung neuer Infrastrukturen und Werkzeuge im Rahmen der wissenschaftlichen Arbeit entsprechende Kenntnisse auf der Anwenderseite wünschenswert sind. Dazu gehört beispielsweise auch ein Grundverständnis für die jeweils genutzten Suchalgorithmen, die den Output von Suchanfragen ganz wesentlich vorgeben. Was und was nicht und aus welchen Gründen Suchanfragen ergeben und wie sich die Ergebnisse auf das jeweilige Forschungsvorhaben sowie auf Schlussfolgerungen zu einzelnen Arbeitspaketen auswirken, darf nicht nur einfach zur Kenntnis genommen, sondern muss auch kritisch begleitet werden.

In den Kapiteln „Lehre und Qualifizierung“ und „Technologien und Dienste“ geht es einerseits um qualifikationsseitige Voraussetzungen, die für erfolgreiches, wissenschaftliches Arbeiten auf dem Gebiet der Digital Humanities zu erfüllen sind, und um das technische Framework, das digitales Arbeiten ermöglicht. Beide Felder sind angesichts der sich permanent wandelnden Herausforderungen an die Forschenden und an die Betreiber von Infrastrukturen von großer Bedeutung. Die Lehr- und Qualifizierungsaktivitäten der F\&E-Abteilung der SUB Göttingen werden am Beispiel der „Qualifizierung im Bereich digitaler Langzeitarchivierung“ von Achim Oswald und Stefan Strathmann dargestellt. Hervorzuheben sind dabei die zahlreichen Kooperationen, die dafür mit vielen und teilweise sehr unterschiedlichen Partnern, insbesondere im Zusammenhang mit NESTOR, dem Kompetenznetzwerk für Langzeitarchivierung, geschlossen wurden. Beachtlich ist die Zahl der Publikationen, die vor allem von Heike Neuroth in Zusammenarbeit mit weiteren Autoren herausgegeben wurden, und die sich unmittelbar dem Thema der Lehre und Qualifizierung auf dem Gebiet der Langzeitarchivierung widmen. Malte Rehbein und Patrick Sahle zeigen in ihrem Beitrag „Digital Humanities lehren und lernen“ auf, wo und in welcher Weise die Digital Humanities mit unterschiedlichen thematischen Schwerpunkten in die Curricula und Studiengänge an Hochschulen eingegangen sind. Dafür werden Beispiele in Deutschland, Europa und über Europa hinaus genannt. Dabei erkennen die Autoren erfreulicher Weise einen wachsenden Bedarf ,an ausgebildeten Wissenschaftlern, die in der Lage sind, die methodische, modellierende, formalisierende und technische Seite der Geisteswissenschaften abzudecken“"(S. 227).

Über universitäre Lehre und die gezielte Qualifizierung auf einzelnen fachlichen Feldern hinaus spielen Informati- onskompetenz und deren kontinuierliche Vermittlung in allen Bereichen von Hochschulen eine wichtige Rolle. Vor dem Hintergrund, dass die digitale Transformation Forschung, Lehre, Studium und Verwaltung auf allen Ebenen verändert und weiter verändern wird, hat die Hochschulrektorenkonferenz (HRK) die Arbeitsgruppe „Zukunft der digitalen Information in Lehre und Forschung“ eingesetzt, deren Empfehlung „Hochschule im digitalen Zeitalter: Informationskompetenz neu begreifen - Prozesse anders steuern“ im November 2012 in Göttingen verabschiedet worden ist. In seinem Beitrag „Hochschule im digitalen Zeitalter. Die Stärkung von Informationskompetenz als Ziel einer Empfehlung der Hochschulrektorenkonferenz" stellt Ulrich Meyer-Doerpinghaus die Genese und das Spektrum dieses Empfehlungspapiers dar. Angesichts der Rolle, die das Thema in allen Bereichen der Hochschule spielt, wird auch der Handlungsbedarf sehr deutlich, der sowohl in entsprechenden Curricula als auch mit geeigneten, oftmals schon bestehenden Kompetenznetzwerken aufgegriffen und der mit wirksamen Angeboten unterstützt werden muss. Von daher setzen die HRK-Empfehlungen auf die Unterstützung bestehender Netzwerke oder die Einrichtung neuer Netzwerke mit spezialisierten Schwerpunkten. Mit dem Appell an Bund und Länder ,ihren Anteil am Aufbau solcher Strukturen zu leisten und entsprechende Maßnahmen zu unterstützen (HRK 2012, S. 18), erklärt sich die HRK zudem bereit, eine Arbeitsgruppe zu gründen, die Kompetenzbildung innerhalb von Fakultäten und Infrastruktureinrichtungen in regelmäßigen Zeitabständen begleitet und Verbesserungen vorschlägt.

Aus guten Gründen ließen sich zu digitalen Diensten und Technologien viele „pralle Blogs“ oder „dicke Bücher“ schreiben und zwar nicht nur aus makrokosmischer Perspektive, sondern durchaus auch mit Blick auf die vielen, technischen, oftmals fast mikroskopisch kleinen Details, deren Bedeutung vor allem dann sehr spürbar ist, wenn der „DATENSTROM“, der den virtuellen Kosmos anreichert und belebt, plötzlich und wider Erwarten zum Stillstand kommt. Von daher waren die Herausgeber des Bandes gut beraten, das Thema der Dienste und Technologien mit Beiträgen zu präsentieren, die - hier kommt der Rezensent auf die Landkarte des Covers der Jubiläumsschrift zurück - internationale und nationale Entwicklungen zum Gegenstand haben, zu denen „DARIAH CENTRAL (D.C.)“, die „GITHUB-Fälle“ und das „Deliverable Delta“ gehören, und die die lokalen Entwicklungen in und um „GRIDopolis“ zusammenfassen und dabei nach Art der „RadieschenPlantage“ und gemäß Anlage der „Wiki-Wiese“ semantisch aufeinander beziehen. $\mathrm{Zu}$ „Forschungsinfrastrukturen in den Geisteswissenschaften" berichten Tobias Blanke und Christiane Fritze und gehen dabei auf „DARIAH-EU 
als europäische Forschungsinfrastruktur" ein. DARIAH ist die „Digital Research Infrastructure for the Arts and Humanities“. In DARIAH-EU, das seit mehreren Jahren mit einem beachtlichen Mittelvolumen gefördert wird, arbeiten viele europäische Länder zusammen. Am deutschen Anteil an DARIAH-EU hat die F\&E-Abteilung der SUB Göttingen wesentlichen Anteil, wie sie selbstverständlich auch DARIAH-DE, dem deutschen Partner von DARIAH-EU, angehört. In dem Beitrag wird auf die organisatorischen und rechtlichen Rahmenbedingungen eingegangen, die erforderlich waren, um DARIAH-EU aus dem Projektstatus heraus in ein europaweites Infrastrukturangebot für die Geistes- und Kulturwissenschaften $\mathrm{zu}$ überführen. Die Community-Dienste werden von sog. Virtual Competency Centers (VCCs) zu übergreifenden Themenkomplexen („einfrastructure“, „research and education“, „scholarly research data“ und „impact, outreach and advocacy“) entwickelt und betrieben. In der Organisationsstruktur von DARIAH-EU sind Vertreter von Infrastruktureinrichtungen und Wissenschaftler vertreten. Koordinationsaufgaben werden zu finanziellen und rechtlichen Entscheidungen von Frankreich, zur Projektkoordination und Öffentlichkeitsarbeit von Deutschland und $\mathrm{zu}$ den virtuellen Kompetenzzentren von den Niederlanden geleistet. In technischer Hinsicht orientiert sich DARIAH-EU an bestehenden und neuen WEB-Technologien, die für einzelne Communities mit einem Schwerpunkt auf dem SocialWeb-Bereich aufgebaut werden.

Aufgrund seiner Bedeutung für die wissenschaftliche Arbeit in virtuellen Forschungsumgebungen passt der Beitrag von Jens Klump und Jens Ludwig zum „Forschungsdaten-Management“ bestens in das Kapitel „Technologien und Dienste“. Die beiden Autoren erklären Aufgaben und Zuständigkeiten im Kontext des Forschungsdatenmanagements und die Rolle, die Bibliotheken dabei einnehmen wollen und können. Dabei stellt sich die Frage, welche konkreten Aufgaben von Bibliotheken beim Forschungsdatenmanagement in Betracht kommen können und für welche - vor allem technische - Dienste in Kooperation mit Partnern der akademischen Informationsinfrastrukturen erbracht werden müssen. Denn Forschungsdatenmanagement ist gerade in naturwissenschaftlichen Disziplinen wie z.B. den Geowissenschaften nicht neu und als Bestandteil von Forschungsvorhaben längst etabliert. Für Bibliotheken verbindet sich der Umgang mit Forschungsdaten mit dem Aufbau neuer Dienstleistungen und Kooperationen und hängt wesentlich damit zusammen, was konkret unter Forschungsdaten verstanden wird und welchem Zweck die im Rahmen des Forschungsdatenmanagements ergriffenen Maßnahmen dienen sollen. Sehr hilfreich ist dafür die - auch anhand einer Tabelle veranschaulichte -
Übersicht zur Rolle der Infrastruktur beim Forschungsdatenmanagement, das organisatorisch im Regelfall nicht lokal und vor Ort, sondern in der Vernetzung lokaler, regionaler, nationaler und internationaler Infrastruktureinrichtungen erfolgt (S. 265). Als eine nicht weniger komplexe Herausforderung erweisen sich oft recht kurze, teilweise auch disruptive Innovationszyklen der informationstechnischen Entwicklungen.

„Digitale Werkzeuge in den digitalen Geisteswissenschaften" werden in dem darauf folgenden Beitrag von Stefan E. Funk, Ubbo Veentjer und Thorsten Vitt vorgestellt, wobei der Status quo und neue Entwicklungen in der virtuellen Forschungsumgebung TextGrid im Mittelpunkt stehen. Dabei wird ein beeindruckendes Spektrum allgemeiner und fachspezifischer Dienste und Tools aufgeführt, die sich auf den gesamten Forschungszyklus beziehen, wie er in TextGrid abgebildet ist. Der modulare Charakter vieler Komponenten und Werkzeuge ermöglicht deren Einsatz und Integration in Arbeitsumgebungen, die außerhalb und unabhängig von TextGrid verfügbar sind. Umgekehrt können in TextGrid auch Tools implementiert genutzt werden, die an anderer Stelle auf entsprechend standardisierter Basis entwickelt wurden. Von daher kommt dieser „Katalog“ digitaler Werkzeuge, der in dem Betrag eingängig, prägnant und zwangsläufig verkürzt dargestellt wird, dem Charakter von „Science Apps“ sehr nahe, indem ein Werkzeugkasten für digitales Arbeiten zur Verfügung steht, der für viele - allerdings nicht für alle - der darin nutzbaren Komponenten keine „monolithische“ Softwarearchitekturen voraussetzt - das ist in der Tat ein großer Gewinn!

Im letzten Beitrage des Kapitels „Technologie und Dienste“ behandeln Peter Wittenburg und Peter Gietz das Thema „Technische Infrastrukturen, Dienste und Services für die digitalen Geisteswissenschaften“. Der Beitrag ist in keiner Weise eine Doppelung zu zuvor behandelten Themen, sondern vertieft eine Reihe von Punkten, die in den vorausgegangenen Beiträgen bereits angesprochen sind. Am Beispiel des Datenmanagements werden die Herausforderungen und Probleme des angemessenen Umgangs mit Daten und der Komplexität ihrer Nutzung dargestellt; ganz ausdrücklich wird dies auf die Geistes- und die Naturwissenschaften bezogen. Auf der Grundlage des vorgestellten Datenmodells, das für die Registrierung und Publikation von Forschungsdaten die Beziehungen zwischen Daten (auf Bitstream-Ebene), persistenten Identifikatoren (PIDs) und Metadaten beschreibt, wird dann auf zentrale Elemente eingegangen, die Infrastrukturen für wissenschaftliches Arbeiten im Kontext von E-Research und E-Science konstituieren. Mit Berücksichtigung ihrer Datenvielfalt, die nun die bereits bestehende Datenvielfalt 
der Naturwissenschaften zu ergänzen beginnt, wird dabei vorrangig von den Geisteswissenschaften ausgegangen. $\mathrm{Zu}$ den zentralen Infrastrukturelementen gehören aus Sicht der Autoren vor allem persistente Identifikatoren, Metadatenbeschreibungen und dafür geeignete Formate sowie verteilte Authentifizierungs- und Autorisierungsstrukturen. Besonders bemerkenswert ist die Forderung nach Registraturen zu persistenten Identifikatoren, Metadatenbeschreibungen, Ontologien einschl. automatisierter Verarbeitungsmöglichkeiten, Schnittstellen für Prozeduren, die in disziplinären Datenzentren für Aggregationsprozesse, Monitoringaufgaben etc. eingesetzt werden, sowie Registraturen für Sammlungen digitaler Inhalte und Schemata, die Recherchen über heterogene Bestände ermöglichen (S. 313f.).

In dem die Jubiläumsschrift abschließenden Kapitel „Ausblick“ geht es um die Weiterentwicklungsperspektiven wissenschaftlicher Bibliotheken und um die geistesund die naturwissenschaftliche Sicht auf das Zusammenwirken von Forschung und Infrastruktur, für das aus gegebenem Anlass die SUB Göttingen und ihre F\&E-Abteilung steht. „Die wissenschaftliche Bibliothek im Kontext von Forschungsinfrastrukturen" ist der Titel des Beitrages von Heike Neuroth. Im ersten Teil des Beitrages wird auf die zahlreichen Empfehlungen und Strategiepapiere auf nationaler und europäischer Ebene eingegangen, in denen wissenschaftlichen Bibliotheken im Zuge der Weiterentwicklung der Informationsinfrastrukturen zu Recht eine Schlüsselrolle zugesprochen wird. Die damit verbundenen Aufgaben finden sich auch im Portfolio der F\&E-Abteilung der SUB Göttingen. Daran anknüpfend wird auf Forschungsinfrastrukturen eingegangen, die in einer Reihe von EU-Projekten (CESSDA, CLARIN, DARIAH, ESS und SHARE) entwickelt und aufgebaut wurden. Aufgrund ihrer spezifischen Ausrichtung auf geistes- und kulturwissenschaftliche Forschungsinfrastrukturen stellt Neuroth das Dienstespektrum von DARIAH-DE als eine eigenständige Infrastrukturentwicklung vor, die konzeptionell in den DARIAH-EU-Kontext eingefügt ist. In DARIAH-DE stehen folgende Schwerpunkte auf der Agenda, die über die jeweiligen Forschungs- und Infrastrukturvorhaben zur weiteren Nachnutzung verfügbar sind: Nachwuchsförderung und Qualifizierungsmaßnahmen, Forschungszyklus und fachwissenschaftliche Dienste, wissenschaftliche Sammlungen und Forschungsdatenmanagement sowie technische Infrastruktur und generische Basisdienste. Im dritten und letzten Teil ihres Beitrags geht Heike Neuroth auf die künftige Rolle wissenschaftlicher Bibliotheken ein (S. 338 ff.). Dazu gehören insbesondere fachwissenschaftliche Informations- und Datenaufbereitung zur Strukturierung und Beschreibung von Informationen und Daten sowie die Bereitstellung fachwissenschaftlicher Informationsdienste und Werkzeuge, um Informationen und Daten verarbeiten und nutzbar machen zu können. Darüber hinaus sind Forschungsdatenmanagement, Langzeitarchivierung, virtuelle Forschungsumgebungen, technische Basisstrukturen sowie Schulungs- und Lehrangebote $\mathrm{zu}$ nennen. Den Ausführungen von Heike Neuroth ist uneingeschränkt zuzustimmen - genau dies sind die Voraussetzungen, um die digitale Transformation in Kooperation mit den Fachdisziplinen voranzutreiben. Ebenfalls zuzustimmen ist ihrer Auffassung, dass die große Herausforderung in der Umgestaltung der Bibliotheken liegt, um die neuen Aufgaben aufzugreifen und in Services umsetzen zu können. Strategiebildung, in- und externe Kommunikation, Personalentwicklung und die Schaffung der notwendigen finanziellen Rahmenbedingungen sind dafür zentrale Voraussetzungen. Mit anderen Worten ließe sich die anstehende Neuausrichtung der wissenschaftlichen Bibliotheken auch in folgender Weise beschreiben: „Not just to display the scholarly information, but to be part of the research process as well!“

Mit ihrem Beitrag „Die wissenschaftliche Bibliothek als Schnittstelle zwischen digitaler Infrastruktur und geisteswissenschaftlicher Forschung “ betrachtet Andrea Rapp das Zusammenwirken von Forschung und Infrastruktur aus geisteswissenschaftlicher Sicht und weist zunächst darauf hin, dass die sich in einem „Definitions- und Verortungsprozess“ befindlichen Digital Humanities in ihrer „digitalen Affiliierung“ so heterogen und vielfältig zeigen, wie dies auch das Spektrum der Geistes- und Kulturwissenschaften ist. Zugleich „reklamierten die Digital Humanities durch den Einsatz computergestützter Verfahren eine entscheidende Erweiterung und Erneuerung der Geisteswissenschaften“ und „versprechen nicht weniger als ein neues Forschungsparadigma“. Der „digital turn“ solle die Geisteswissenschaften verändern und einen „empirical turn" bewirken (S. 346f.). Entscheidend ist dabei für Rapp, dass die „Explizierung vom impliziten Wissen, die Möglichkeiten des Zugriffs auf Massendaten bzw. auf größere Quellen- und Datenumfänge und die ,Strenge“ (rigor) des Arbeitens, die der Computereinsatz uns abverlangt“ oftmals neue und äußert vollständig kartierte Wissensräume zur Erforschung neuer Fragestellungen bieten. Als Wissenschaften des Diskurses und Verstehens seien den Geisteswissenschaften damit eine Verbreiterung der bisher zugänglichen Datengrundlage und eine „Explizierung des in unseren Daten- und Wissensspeichern inhärenten Wissens“ verfügbar, was - „verbunden mit einer gewissen Annäherung an naturwissenschaftliche Zugänge“ - für ihre Forschungsfragen gewinnbringend sei, ohne dabei etwas aufgeben zu müssen. Von daher verbinden 
sich mit dem Zugriff auf bisher nicht zugängliche Quellen oder einfach nur „mehr“ Quellen für die Geisteswissenschaften neue und eben „mehr“ Erkenntnisse. In Anbetracht dessen wird in der Zusammenarbeit zwischen digitaler, geisteswissenschaftlicher Forschung und wissenschaftlichen Bibliotheken, deren Tradition auf der kontinuierlichen Weiterentwicklung der Kulturtechniken des Bewahrens und Erschließens beruht, die große Chance einer für beide Seiten Nutzen bringende Evolution und Weiterentwicklung gesehen. Diese Sicht auf die Weiterentwicklung der Geisteswissenschaften und der damit verbundenen Aufgaben der Bibliotheken beantwortet die Leitfrage der Jubiläumsschrift mit einem hohen Maß an Ausgewogenheit und umsichtiger Reflexion.

Abschließend kommt mit dem Beitrag von Arnulf Quadt eine Sicht der Naturwissenschaften zu Wort: „GridComputing in der Teilchenphysik, Fortschritt durch Technik“. Am Beispiel der Teilchenphysik wird aufgezeigt, dass Verbesserungen von Infrastruktur und Methoden der Forschung Möglichkeiten und Voraussetzungen für neue Einblicke und Erkenntnisse bieten. Das Beispiel der Teilchenphysik ist deshalb bestens gewählt, weil die Korrelationen zwischen datengetriebener Forschung und skalierender Infrastruktur im Hinblick auf Erkenntnisdurchbrüche und Forschungserfolge sehr sichtbar werden. Beispielhaft dafür ist der Entwicklungsstand des Large Hadron Collider (LHC) des CERN und dessen Experimente, die bisher unbekannte Elementarteilchen und Prozesse entdecken wollen, und an denen die Universität Göttingen mit dem ATLASProjekt beteiligt ist. „Diese Art der Forschung gleicht buchstäblich der Suche nach der Nadel im Heuhaufen“, schreibt Quadt. Heute seien die Auswertungen der aufgezeichneten Teilchenkollisionen, die zu früheren Zeiten manuell erledigt wurden, nur noch mit weltweit verteilter Datenspeicherung und der Nutzung in gleicher Weise verteilter CPU-Ressourcen möglich (S. 358). An der aus dem GRID-Konzept entstandenen Projekte MediGrid und TextGrid war und ist die F\&E-Abteilung der SUB Göttingen unmittelbar beteiligt und damit auch an der Entwicklung des GoeGrid-Clusters, mit dem die Universität Göttingen nationale und internationale Sichtbarkeit erreicht und im Zeitraum 2010-2012 einen signifikanten Beitrag zur Analyse von LHC-Daten geliefert hat. Mit dem Hinweis, dass von den Experimenten ATLAS und CMS am 4. Juli 2012 die Entdeckung eines neuen Bosons verkündet wurde, das sich ,in allen bisher untersuchten Eigenschaften als kompatibel mit dem seit 1964 gesuchten Higgs-Boson darstellt", dankt Arnulf Quadt der F\&E-Abteilung und wünscht ihr weiterhin allen Erfolg.

Any further questions? Ja, die Allerweltsfrage, die sich in Zeiten von Wandel und Veränderung stellt: Was gewin- nen wir mit der digitalen Transformation und was geht möglicher Weise verloren? Wie immer ist diese Frage vergeblich, weil ihre Beantwortung sich üblicherweise nicht aus dem Wandel ergibt, und der Wandel selbst sie nicht beantworten kann. Zudem ist die Frage falsch gestellt, weil ein ultimatives „Entweder-Oder“ gar nicht gegeben ist. Wissenschaftliches Arbeiten kennt disziplinspezifische Herangehensweisen und Methoden. Doch das heißt nicht, dass sich verschiedene epistemische Ansätze gegenseitig ausschließen müssen. Vielmehr ist $\mathrm{zu}$ beobachten, dass sich unterschiedliche Forschungsformen oftmals wechselseitig ergänzen. Mit dem erweiterten Materialspektrum, das sich den Geisteswissenschaften mit digitalen Verfahren erschließt, geht deshalb nichts verloren, sondern es stellen sich alte Fragen neu, und neue Fragen lösen alte Fragen ab. Zugleich treten Bibliotheken und Wissenschaft in eine Kooperation, die eine neue interdisziplinäre Qualität erreicht und die für die Weiterentwicklung beider Partner äußerst gewinnbringend ist. $\mathrm{Zu}$ adressieren ist allerdings, dass bewusst gewusst werden sollte, was man mit digitalen Infrastrukturen und Verfahren tatsächlich macht. Doch dergleichen fordert schon die Neugier am eigenen Handeln - über die dafür notwendige Informationskompetenz verfügt die Bibliothek.

In der 3D-Verfilmung des Comics „The Guardians of the Galaxy" ist einer der vier Protagonisten ein Baum, der über die beiden folgenden Stärken verfügt. Zum einen wendet er Gefahren von seinen Mitstreitern ab, indem er seine Äste zu einem Netz verformt und so die ihm Anvertrauten schützend umschließt. Zum anderen sind seine Äste so regenerationsbereit, dass im Fall seines Verendens sofort die nächste Generation seiner Gattung aus dem Boden sprießt. Indem dieser Baum in anderen Umgebungen aufgeht, entsteht er neu - das ist Nachhaltigkeit, wie sie auch für Bibliotheken kennzeichnend ist, die künftig möglicher Weise TextGrid heißen. Und wie nennt sich der Baum der „Guardians of the Galaxy“? „My name is Groot“, lässt uns die Stimme von Vin Diesel immer wieder aufs Neue wissen.

Mit der Jubiläumsschrift anlässlich des zehnjährigen Bestehens der F\&E-Abteilung der SUB Göttingen wird ein Meilenstein dokumentiert und zugleich ein profunder Überblick zum Stand und zur Weiterentwicklung digitaler Infrastrukturen insbesondere in den Geisteswissenschaften und zu der sich wechselseitig befruchtenden Kooperation zwischen Bibliothek und Forschung gegeben. Ein umfassendes Themenspektrum wird mit Beiträgen ausgewiesener Experten aus unterschiedlichen Sichten präsentiert und ist dabei mit einer Fülle von Literaturangaben im unmittelbaren Anschluss an die Beiträge hervorragend „kontextualisiert“. Ausgangspunkt des Bandes ist die SUB 
Göttingen, doch im Ergebnis geht es um die Voraussetzungen und Herausforderungen für erfolgreiches, digitales Arbeiten in den Geisteswissenschaften - für Akteure auf dem Gebiet der „Digital Humanities“ ist die Lektüre dieser Jubiläumsschrift deshalb zweifellos Pflicht!

\section{Umlauf, Konrad; Fühles-Ubach, Simone; Seadle, Michael} (Hg.): Handbuch Methoden der Bibliotheks- und Informationswissenschaft. Bibliotheks-, Benutzerforschung, Informationsanalyse. Berlin: De Gruyter Saur, 2013 (Reference). 561 S. ISBN 978-3-11-025553-9. 149,95 EUR

Besprochen von Prof. Dr. Hans-Christoph Hobohm: Professor für Bibliothekswissenschaft, Fachbereich Informationswissenschaften, Fachhochschule Potsdam, Friedrich-Ebert-Str. 4, D-14467 Potsdam, E-Mail: hobohm@fh-potsdam.de

Nach der Fortsetzung des Busse/Ernestus durch Plassmann, Rösch u. a., dem „Handbuch Bibliothek“ im Metzler Verlag und dem „Lexikon der Bibliotheks- und Informationswissenschaft" bei Hiersemann durch Umlauf und Gradmann ${ }^{1}$ ist das zu besprechende Buch ein weiterer Baustein für eine Bibliothekswissenschaft initiiert durch den einzigen bibliothekswissenschaftlichen Lehrstuhl in Deutschland und in umfangreicher personeller Zusammenarbeit mit dem Kölner Institut für Informationswissenschaft der dortigen FH. Nach dem theoretisch-deskriptiven und dem terminologischen folgt folgerichtig das methodologische Rüstzeug für das immer noch junge oder zumindest „Kleine Fach“. Folgerichtig ist auch im Sinne des Instituts-Namens die zunehmende Hereinnahme der Informationswissenschaft entsprechend der „Library and Information Science (LIS)“ unter der das Fach im Angloamerikanischen seit langem firmiert.

Es erstaunt allerdings, dass in Deutschland unter dem methodologischen Fokus erst jetzt gleich mehrere Publikationen erscheinen. ${ }^{2}$ International ist „Methode“ der Bibliothekswissenschaft seit über einem viertel Jahrhundert ein

\footnotetext{
1 Plassmann, Engelbert; Rösch, Hermann; Seefeldt, Jürgen; Umlauf, Konrad (2011): Bibliotheken und Informationsgesellschaft in Deutschland. Eine Einführung. 2. Aufl. Wiesbaden: Harrassowitz. Umlauf, Konrad; Gradmann, Stefan (Hg.) (2012): Handbuch Bibliothek. Geschichte, Aufgaben, Perspektiven. Stuttgart: Metzler. Gradmann, Stefan; Umlauf, Konrad (Hg.) (2010-2014): Lexikon der Bibliotheks- und Informationswissenschaft. (LBI). 2 Bände. Stuttgart: Hiersemann.

2 Siegfried, Doreen; Nix, Sebastian Johannes (2014): Nutzerbezogene Marktforschung für Bibliotheken. Eine Praxiseinführung. Berlin/Boston: De Gruyter Saur (Praxiswissen).
}

wichtiger Publikationszweig ${ }^{3}$, der rege bedient wird. ${ }^{4}$ Auch andere Fächer wie die Museologie ${ }^{5}$ und die (,praktische“) Informationswissenschaft ${ }^{6}$ weisen teilweise schon lange Traditionen methodologischer Überlegungen auf.

Dafür liegt nun ein 560 seitiges Werk vor, das als Standardwerk für die deutsche Bibliotheks- und Informationswissenschaft wird dienen können. In der Einleitung wird zunächst der Methodenbegriff verdeutlicht und darauf hingewiesen, dass das Handbuch eine Einführung in das wissenschaftliche Arbeiten nicht ersetzen kann. Dennoch wird eine didaktische Lektüreanleitung gegeben, die in diesem Sinne interpretiert werden kann: Beginnend mit Hinweisen zur Erstellung von Literaturbericht (Umlauf, S. 25-40) und Forschungsdesign (Seadle, S. 41-63) sowie der Gegenüberstellung von quantitativen (Fühles-Ubach u. Umlauf, S. 80-95) und qualitativen Methoden (Rinsdorf, 64-79) wird dem Leser empfohlen, sich die für sein eigenes Forschungsvorhaben geeigneten Methoden genauer anzusehen. In den ersten Kapiteln wird wiederholt explizit betont, dass Bibliotheks- und Informationswissenschaft empirisch, wissenschaftlich vorgeht. Ohne es explizit zu erwähnen, folgt Seadle z. B. hierbei dem Konzept des Evidence Based Library and Information Practice ${ }^{7}$ und dem den Naturwissenschaften entlehnten Vorgehen der sich aufbauenden Forschungsfront. Unter der Thematik „Forschungsdesign“ wird hierbei besonders Wert gelegt auf Hypothesenbildung und Datengrundlage einer Untersuchung. Auch wenn Foucault, Geertz und Latour erwähnt werden, positioniert sich die Berliner Informationswissen-

3 Connaway, Lynn Silipigni; Powell, Ronald R. ([1988] 2010): Basic Research Methods for Librarians. 5. Aufl. Santa Barbara, Calif.: Libraries Unlimited (Library and information science text series). McClure, Charles R.; Hernon, Peter (Hg.) (1991): Library and Information Science Research. Perspectives and Strategies for Improvement. Norwood, N.J.: Ablex Pub. Corp (Information management, policy, and services).

4 Um nur wenige der Wichtigeren zu nennen: Baker, Linda M. (Hg.) (2006): Research Methods. Special Issue of "Library Trends". Vol. 55,1. Wildemuth, Barbara. M. (2009): Applications of Social Research Methods to Questions in Information and Library Science. Westport, Conn: Libraries Unlimited. Goodman, Valeda Dent (2011): Qualitative Research and the Modern Library. Oxford [u.a.]: Chandos. Pickard, Alison Jane; Childs, Sue (2013): Research Methods in Information. 2. Aufl. London, Chicago: Facet; Neal-Schuman.

5 Baur, Joachim (Hg.) (2010): Museumsanalyse. Methoden und Konturen eines neuen Forschungsfeldes. Bielefeld: Transcript (Kulturund Museumsmanagement).

6 Kuhlen, Rainer; Semar, Wolfgang; Strauch, Dietmar (Hg.) (2013): Grundlagen der praktischen Information und Dokumentation. 6. Aufl. Berlin: De Gruyter Saur.

7 Booth, Andrew; Brice, Anne (Hg.) (2004): Evidence-Based Practice for Information Professionals. A Handbook. London: Facet Publishing. 
schaft damit wissenschaftstheoretisch eindeutig im kritischen Rationalismus, aber anders als in der empirischen Sozialforschung üblich, wird kein Bezug genommen auf den Kreislauf zwischen Problemauswahl, Theoriebildung, Operationalisierung und Datenanalyse, der die Auswahl der Untersuchungsmethode bestimmt. ${ }^{8}$

Interessanterweise folgt als nächstes Kapitel, vielleicht als Kontrast gedacht, die Darstellung „Qualitative[r] Methoden“, bei der Rinsdorf sehr anschaulich in die komplexe Argumentation der empirisch orientierten qualitativen Sozialforschung von ihren erkenntnistheoretischen Implikationen über den Grounded-Theory-Ansatz bis hin zur Triangulation der Methoden einführt. Er schließt sein Kapitel dennoch mit dem Plädoyer für eine „kreative Kombination qualitativer und quantitativer Methoden“, weil im „Verwendungszusammenhang [...] der Betriebswirtschaft eine Quantifizierung von Befunden gewünscht wird, um sie anschlussfähig zu Markt-, Erlös- oder Ergebnisprognosen zu machen“ (S. 78). Eingangs des folgenden Kapitels „Quantitative Methoden“ wird noch einmal kurz grundlegend auf den Unterschied zwischen qualitativem, auf das individuelle Verstehen ausgerichteten Ansatz, im Vergleich $\mathrm{zu}$ quantitativen Verfahren eingegangen, um auch hier noch einmal deutlich die Nähe der Bibliothekswissenschaft zur Betriebswirtschaft, Marktforschung und Marketing hervorzuheben: „[q]antitative Daten sind daher gut geeignet, um einen Überblick über bspw. Nutzerwünsche zu erlangen“ (S. 91). Vor diesem Hintergrund geben Fühles-Ubach und Umlauf in diesen Kapitel einen knappen Überblick zu grundlegenden Begrifflichkeiten der empirischen Sozialforschung (z. B. u. a. Nullhypothese, Reliabilität, Sekundärforschung, Beobachtung, Experiment, Panel etc.) und bieten erste Hinweise zu ihrem Einsatz in der Bibliothekswissenschaft unter Hervorhebung von drei Instrumenten, deren Einordnung und Methodik an dieser Stelle nicht diskutiert wird: Liqual+ $\left(\right.$ sic $\left.^{9}\right)$, Bibliotheksindex und ,kontingente Bewertungsmethode“ (S. 87f.). Das Kapitel endet richtigerweise mit dem Verweis auf vorhandene Skalen der Kommunikations- und Marktforschung sowie einer kritischen Reflexion der Validität von Kausalitätsaussagen. Es folgen zwei informative Kapitel von Fühles-Ubach zu quantitativen (S. 96-113) und zu Online-Befragungen (S. 114-127), in denen bei aller sonstigen

8 Schnell, Rainer; Hill, Paul B.; Esser, Elke (2011): Methoden der empirischen Sozialforschung. 9. Aufl. München: Oldenbourg, S. 4; vgl. auch Flick, Uwe; Kardorff, Ernst von; Steinke, Ines (Hg.) (2012): Qualitative Forschung. Ein Handbuch. 9. Aufl. Reinbek bei Hamburg: Rowohlt-Taschenbuch-Verlag (Rororo Rowohlts Enzyklopädie, 55628).

9 Gemeint ist: LibQUAL+®. knappen Korrektheit manche (veraltete?) Terme und Einzelhinweise auffallen („Repräsentanz“ (statt „Repräsentativität“), „WWW-Befragung“, MMS, „WAP (Wireles Access Point)“ statt ,[...] Application Protocol“).

In dem Kapitel „Qualitative Befragungen“ (S. 128-151) räumt Werner zunächst mit dem Vorurteil auf, der qualitative Ansatz brauche keine methodische Stringenz und weist daraufhin, dass die praktische Umsetzung dessen, was unter dieser Flagge läuft, ,im Vergleich zum theoretischen Anspruch“ oft zu wünschen übrig lässt (S. 129). Kenntnisreich werden Vorgehen und einzelne Techniken der qualitativen Befragung (teilstrukturiertes Interview, fokussiertes Interview, narratives Interview, Experten-Interview und Gruppendiskussion) beschrieben. „Fallstudien“ (S. 152-167) skizziert Mertes im Anschluss als Sonderfall zwischen den Richtungen. Ihre Beispiele aus dem Bibliothekarischen erscheinen aber eher als Anwendungen von methodologischem Pluralismus (,mixed method“) denn als genuine Fallstudien (es sei denn man versteht unter „Case“ eine bestimmte Zielgruppe).

Etwas aus dem Rahmen fällt der Abschnitt Clusterund Diskriminanzanalyse von Galliat (S. 168-183), der praktisch als Einziger Datenanalyse und statistische Auswertungspraxis in den Blick nimmt. Auch wenn hier wieder ein rein Marketing orientierter Ausgangspunkt gewählt wird („Methoden, die [...] zur Identifizierung prospektiver personalisierter Angebote genutzt werden können“ (S. 168)), erschließt sich nicht ganz, warum einerseits einfachere Auswertungsverfahren wie Randauszählungen, Mittelwertvergleiche oder andere komplexe Verfahren wie Regressionsanalysen oder Faktoren- und Korrespondenzanalyse im ganzen Band unerwähnt bleiben. Im Gegensatz zu anderen Kapiteln erhält der Leser hier sehr tiefgehendes Spezialwissen. Ebenfalls spezialisiert, aber etwas näher an der bibliothekarischen Praxis ist Schlögls Kapitel zu „Logfile und Link-Analysen“" (S. 184-202). Hier werden Einsatzmöglichkeiten nichtreaktiver Verfahren der Onlineforschung von allgemeinen Webanalytics und eRessourcenNutzungsmessung (wie z.B. COUNTER) bis hin zu weitergehenden Netzwerkanalysen anschaulich dargestellt. Ergänzend dazu stellt Richter „Methoden der Usability-Forschung“ (S. 203-256) recht ausführlich und teilweise redundant vor. Dabei konzentriert er sich auf die Methode des Cognitive (hier genannt ,heuristisches') Walkthrough und des Thinking Aloud und behandelt komplexere Verfahren wie z. B. Eye-Tracking, experimentelle Settings oder Methoden aus der HCI-Forschung oder dem UX Design nicht. Letzterem kommt Greifeneder in ihrem sehr stringenten Kapitel „Benutzerforschung“ (S. 257-283) dann näher. Vom Forschungsdesign, über das Erhebungssetting, Fragebogendesign und Auswertungspraxis bis zur recht- 
lichen und institutionellen Einbettung behandelt der Abschnitt in nuce alle Phasen und Aspekte bibliotheks- und informationswissenschaftlicher Forschung mit konkreten Nutzern. Breiter angelegt ist das Kapitel von Umlauf zu "Methoden der Marktforschung und Bedarfsanalyse“ (S. 284-314). Anders als der Titel es suggeriert, bleibt dieses allerdings vorwiegend bei Marktforschung und kommt nicht wirklich zu Bedarfsanalyse, zumindest im Sinne einer Informationsbedarfsanalyse für Organisationen ${ }^{10}$, die z. B. für Spezial- und Unternehmensbibliotheken wichtig wären. Die diskutierten Marktforschungsmethoden reichen bis weit in die Methoden des Qualitätsmanagements hinein, u. a. mit der Darstellung von Blueprinting und SEM (sequentielle Ereignismethode), ohne dass deren Bezug zu Prozessanalyse oder anderen Formen der Sequenzanalyse aufgezeigt werden. Trotz seiner Länge macht das Kapitel deutlich, dass die Methoden der Bibliotheks- und Informationswissenschaft so vielfältige Anleihen aus anderen Methodologien machen, dass deren Darstellung in kondensierter Form nur Ausschnitt- und Anregungscharakter haben kann.

So scheinen auch die folgenden Kapitel jeweils sehr spezifische Aspekte zu behandeln. Der mit „Ethnomethodologie“ überschriebene Abschnitt (Seadle, S. 315-337) behandelt vor allem ethnologische Methoden im Sinne von Clifford Geertz' thick description und vertieft die in vorhergehenden Kapiteln erwähnten qualitativen Erhebungsmethoden auch mit internationalen Beispielen. Von „Ethnomethodologie“ im eigentlichen Sinn (phänomenologische Praxis- und Alltagsforschung oder gar Konversationsanalyse nach Garfinkel und Sacks) ist hier leider nicht die Rede. Geertz selber betonte immer wieder, dass seine ,dichte Beschreibung، nicht als Technik oder Forschungsmethode missverstanden werden sollte. Die „Methoden der Informetrie“ stellt Havemann (S. 338-367) umfassend und konzise dar, ist dies ja auch lange Zeit einer der Schwerpunkte der Berliner Bibliothekswissenschaft gewesen. Auch das Kapitel „Methoden der Evaluation von Informationssystemen“ (Petras, S. 368-386) stellt für diesen Zweig der Informationswissenschaft eine gute Einführung dar, obwohl sich im Kontext der anderen Kapitel naturgemäß auch Redundanzen ergeben (thinking aloud, usability etc.). Unter Informationssystemen werden in diesem Fall deutlich vorwiegend technische Systeme verstanden, die Evaluation von Organisationen, Prozessen oder

10 Dorner, Daniel G.; Gorman, Gary E.; Calvert, Philip J. (2011): Information Needs Analysis. Principles and Practice in Information Organizations. London: Facet Publishing.
Objekten im Sinne des Qualitätsmanagement etwa kommt hier (berechtigterweise) nicht in den Blick.

Tiefer in die „Grundbausteine der Information“ geht Coy bei der Darstellung von „Modellierung und Tests“ (S. 387-397), wobei er leider nicht sehr konkret wird, wie Modellierungs- und Testverfahren ablaufen und welche es gibt. „Juristische Methoden und Arbeitstechniken“ stellt Steinhauer dar (S. 398-411), der betont, dass der Ansatz juristischer Normierungsarbeit auch der Bibliothekswissenschaft zugrunde liegt, etwa bei der Entwicklung von Regelwerken und dass diese damit von entsprechenden Methoden des anderen Faches (wie z. B. der historischgenetischen Auslegung) profitieren kann. Rechtstheoretische oder ethische Frageansätze behandelt Steinhauer an dieser Stelle nicht. Volpers beschreibt im folgenden Kapitel die kommunikationswissenschaftlich geprägte qualitative „Inhaltsanalyse“ (S. 412-424). Obwohl es in der Informationswissenschaft nahe liegen würde, werden die klassischen automatisierten Verfahren (z. B. die aktuell sehr verbreitete Methode der sog. „Wordles“) oder spezifische Methoden des corpusbasierten Tagging von elektronischen Texten nicht behandelt (z. B. General Inquirer oder TEI).

Bei aller begrifflichen Problematik bleibt R. Keller im Kapitel „Diskursanalyse“ (S. 412-443) erfreulich neutral und entfaltet das komplexe für die Bibliotheks- und Informationswissenschaft fruchtbare Feld, das er eher auf den Foucault'schen, kritischen Diskursbegriff bezieht und von der Diskurslinguistik abgrenzt. Hilfreich ist hierbei auch die konkrete Darstellung einer diskursanalytischen Vorgehensweise im engeren Sinn (Forschungsdesign, Datenanalyse und -aggregation). A. Keller konstatiert im Abschnitt „Delphi-Methode“ (S. 444-460) zunächst die Beliebtheit dieses Instruments, die sie mit der Überschaubarkeit des Aufwands begründet, obwohl sie später darauf hinweist, dass die meisten Delphi-Studien im LIS-Bereich Dissertationen waren. Die breiter angelegten Delphi-Studien zur Informationsgesellschaft (z. B. die Zukunftsstudien des Münchner Kreises oder der Horizon Report) kommen nicht in den Blick des Kapitels, wie überhaupt Methoden der Zukunftsforschung in dem besprochenen Band interessanterweise kaum angesprochen werden.

Stattdessen finden sich zwei Kapitel zu eher historischen Methoden von namhaften Autoren: die historische „buchwissenschaftliche Forschung“ (Rautenberg, S. 461-482) und „historische Bibliotheksforschung“ (Mittler, S. 483-524). Beides sind für den an der Thematik Interessierten äußerst lesenswerte Texte, die zeigen, dass andere Disziplinen in der Tat ein methodisch-theoretisches Instrumentarium aufgebaut haben, das bibliothekswissenschaftlich genutzt werden sollte. Den Abschluss des Bandes bildet ein unter der gleichen Perspektive sehr 
aufschlussreiches Kapitel zur modernen „Lese- und Leserforschung“ (Rühr, Mahling, Kuhn, S. 525-546).

Fast alle Kapitel weisen ein ausführliches eigenes Literaturverzeichnis auf, so dass es kein Gesamtquellenregister gibt. Das 14-seitige kombinierte Sach- und Personenregister ist hilfreich für den Einstieg in einzelne Bereiche, ersetzt aber nicht ein denkbares Glossar, der für den Neuling manchmal komplexen und heterogen gebrauchten Begrifflichkeiten.

So sehr die Berlin-Kölner Initiative zu begrüßen ist, umso mehr erstaunt insgesamt der geringe Bezug zu Nachbarwissenschaften und international Erreichtem (Ausnahmen bestätigen die Regel). In den meisten der 24 Kapitel wird der gleiche Transfer sozialwissenschaftlicher Methoden in das eigene Fach betrieben wie in vielen anderen Disziplinen. Die Spezifik ihres Einsatzes im Bibliotheksund Informationsfeld wird zwar immer wieder versucht, wobei die Texte dann oft weder der Forschungs-Methode noch dem Praxis-Problem gerecht wird. Ebenfalls fällt auf, dass der Bezug zwischen Theorie, Wissenschaft, Methode und der Praxis nicht explizit diskutiert wird, wie dies z.B. anschaulich Marcia Bates im Kontext der Informationsverhaltensforschung tut. ${ }^{11}$ So gibt es recht wenig meta-theoretische Reflexion zu den jeweiligen vorgestellten Methoden in ihrem paradigmatischen Kontext. Steht doch keine Methode in einem luftleerem Raum und ist immer bedingt durch ihre jeweilige wissenschaftliche Grundhaltung: Die langen methodologischen Diskussionen in anderen Disziplinen wie etwa der Geschichtswissenschaft, der Wirtschaftswissenschaft aber auch der Sozialforschung selber stehen stets unter der Frage des Verdachts festgelegter Denkrichtungen, die die jeweilige andere Schule nicht akzeptiert. Von „Methodenstreit“, ja von Methodenreflexion vielleicht auch nur in wissenschaftstheoretischem Sinn, ist in dem Buch wenig zu finden. Der Leser hat meist eher den Eindruck, dass alles genauso ist, wie es beschrieben wird. Es entsteht das Bild einer kontextlosen praktischen Anleitung zur Anwendung sozialwissenschaftlicher Methoden in einem bestimmten Gebiet, ohne dass das Gebiet definiert würde und eher diffus und austauschbar bleibt. Die Methoden hätten jede andere Einrichtung betreffen können. Manche eher informationswissenschaftliche oder spezifisch sozialwissenschaftliche Methoden werden berechtigterweise aus Raumgründen ausgeblendet und die Auswahl nicht begründet. Das scheint vielleicht der in dem Buch vertretenen Position einer Bibliotheks-

11 Bates, Marcia J. (2005): An Introduction to Metatheories, Theories, and Models. In: Fisher, Karen E.; Erdelez, Sanda; Mckechnie Lynne (Hg.): Theories of Information Behavior. Medford, N.J.: Information Today (ASIST monograph series) S. 1-24. wissenschaft als empirischer Handlungswissenschaft und der Bibliothek als betriebswirtschaftliche Einheit geschuldet. Vielleicht ist die Bibliothekswissenschaft doch eher eine Reflexionswissenschaft und insbesondere die Informationswissenschaft näher an den Behavioral Economics als an klassischer BWL und empirischer Sozialforschung. Insofern ersetzt das Buch den Verweis auf die eingangs erwähnten Handbücher aus dem Ausland und den Urdisziplinen der Sozialforschung leider nicht.

\section{Où en est l'histoire des bibliothèques? Dossier publié} sous la direction de Frédéric Barbier. Genève: Droz, 2014. $488 \mathrm{~S}$. s/w. Abb. (Histoire et civilisation du livre: revue internationale; 10). Brosch. ISBN 978-2-600-01840-1, ISSN 1661-4577. € 40,-

Besprochen von Prof. Dr. Peter Vodosek: Seestraße 89, D-70174 Stuttgart, E-Mail: vodosek@hdm-stuttgart.de

Die Librairie Droz in Genf verlegt seit 1924 wissenschaftliche Literatur mit dem Schwerpunkt auf Mittelalter- und Humanismusstudien, Literaturkritik, Kunst- und Buchgeschichte sowie Sozialwissenschaften. 2005 erschien der erste Band der internationalen Fachzeitschrift „Histoire et civilisation du livre“. Ihre Erscheinungsweise ist jährlich, so dass man auch von einem Jahrbuch sprechen könnte. Jeder Jahrgang bringt ein oder mehrere „Dossiers“, Sammlungen von Aufsätzen zu einem speziellen Thema. Band 10 (2014) enthält die Dossiers „Où en est l'histoire des bibliothèques?“ und „Études d'histoire du livre“ sowie einen Einzelbeitrag „Livres, travaux et rencontres“. Herausgeber der Zeitschrift wie auch dieses Bandes ist Frédéric Barbier, einer der renommiertesten Buchwissenschaftler Frankreichs. Er wirkt als „Directeur de recherche“ am „Centre national de la recherche scientifique (CNRS)/ Institut d'histoire du monde contemporain/École normale supérieure ULM“ und als „Directeur d'études à l'école pratique des hautes études (EPHE)“. Zuvor war er auch an der seinerzeit in Göttingen ansässigen „Mission historique française en Allemagne“ und am „Centre Gabriel Naudé/ Centre de recherches en histoire du livre“ der „École nationale supérieure des sciences de l'information et des bibliothèques (ENSSIB)“ in Villeurbanne tätig.

Anders als in Deutschland wird in Frankreich die Bibliotheksgeschichte schon seit langem als ein wesentlicher Teil der Buch- und Ideengeschichte betrachtet (möglicherweise ist dies auch der Grund, warum der Band keinen Beitrag von deutscher Seite enthält). Es ist daher nicht weiter verwunderlich, dass der Anstoß von französischer Seite kam, mit einer Reihe von Beiträgen zu dokumentie- 
ren, wo die Bibliotheksgeschichte heute steht. Das Dossier „Où en est l'histoire des bibliothèques?" soll daher in den Mittelpunkt dieser Rezension gerückt werden.

Lange Zeit, so die Überlegungen, die zur Publikation dieses Bandes geführt haben, sei die Geschichte der Bibliotheken vornehmlich unter zwei Gesichtspunkten behandelt worden: einmal von den Beständen her; dann als Geschichte von Institutionen, gewissermaßen als die Geschichte von Individuen. Diese Art von Geschichtsschreibung habe zum Teil etwas von Hagiographie an sich gehabt. Seit einigen Jahren aber hätten sich neue Ansätze gezeigt und neue Themen ergeben, nicht zuletzt unter dem Einfluss der sich rapide ausbreitenden neuen Medien. Exemplarisch zu nennen wären die Bibliothek als Raum, die Geschichte des Lesers als Ergänzung der Geschichte des Lesens, die Politik der Bibliothek und nicht zuletzt die Probleme des Kulturtransfers. Perspektiven der Sozialgeschichte entwickelten sich nach und nach zur Denkweise einer historischen Anthropologie. Auf diese und andere Fragen geht Barbier als Herausgeber in seinen einleitenden Bemerkungen ein. Für ihn war es nicht das Anliegen, in einer Nummer dieser Zeitschrift die Fülle der Probleme auszubreiten. Vielmehr sollte eine Auswahl von Beispielen aufzeigen, was Bibliotheksgeschichte im Licht neuer Blickweisen heute sein könnte. Damit verbindet er die Hoffnung, dass der Band dazu beitragen werde, den Horizont zu erweitern.

Die 13 Beiträge des Dossiers stammen, einer „Revue internationale“ angemessen, von Autoren aus sechs Ländern: Frankreich (5), Italien (4), der Schweiz, Portugal, Ungarn und Brasilien (je 1). Zwei Texte sind in italienischer Sprache. Die Beiträger kommen aus dem Umfeld von Universitäten und anderen Forschungseinrichtungen (z. B. von EPHE), vier von ihnen aus Bibliotheken. Einige Texte gehen auf akademische Abschlussarbeiten zurück. Gut die Hälfte der Beiträge beschäftigt sich mit dem weiten Feld der Privat-(Gelehrten-, Adels- usw.)Bibliotheken vom 15. bis zum 19. Jahrhundert.

Georges Bischoff, Professor an der Universität Straßburg, spricht von einem „Printemps du livre“ mit Straßburg und dem Oberrhein als Zentrum, zeitlich eingeordnet zwischen dem Konzil von Basel (1431 und 1448/1449) und dem Wirken von Sebastian Brant (†1521). Das Konzil war im Vor-Gutenberg-Zeitalter eine „Börse für Bücher“. Für das dritte Viertel des 15. Jahrhunderts hebt Bischoff die Bedeutung des Klosters Murbach und seiner Bibliothek unter dem Abt Georg von Andlau, dem ersten Rektor der Universität Basel, hervor. Mit der Erfindung des Buchdrucks setzt eine rapide Entwicklung der Privatbibliotheken ein, deren Genese im adeligen und bürgerlichen Milieu noch viele Fragen offen lässt (S. 13-26). Der italienischsprachige Beitrag von Giancarlo Petrella (Katholische Uni- versität Mailand-Brescia) gewährt einen ersten Einblick in eine Adelsbibliothek. Es handelt sich um die Bibliothek der Grafen Thun auf Castel Thun (Ton) in Vigo di Ton im Trentino. Sie war eher eine nicht planmäßig zustande gekommene Sammlung aus dem Besitz von verschiedenen Familienmitgliedern, die in einem Zeitraum von fünf Jahrhunderten entstand. Um ihre ursprüngliche Gestalt zu rekonstruieren sind weitere Nachforschungen erforderlich (S. 27-50). Magali Jacquinez (Universität Straßburg) geht S. 131-141 den Ursprüngen der alten Stadtbibliothek Straßburg nach, die 1754 nach einem Vermächtnis von Johann David Schoepflin (1694-1771) gegründet worden ist, der seine Sammlung der Stadt hinterlassen hat. Sie ist bekanntlich im Deutsch-Französischen Krieg im August 1870 durch Artilleriebeschuss zerstört worden. Ein bisher unbekanntes Dokument vom 6. Juni 1650 wirft ein neues Licht auf die Vorbilder und juristischen Schritte, die zur Gründung einer für die Öffentlichkeit bestimmten Bibliothek durch den Kardinal Mazarin geführt haben: Yann Sordet, Direktor der Bibliothèque Mazarine, beleuchtet die Vorgeschichte, die eng mit dem Namen Gabriel Naudé verbunden ist, und bindet sie in die politischen Ereignisse ein (S. 93-111). Antonella Barzazi (Universität Padua) verfolgt den Weg von der Gelehrten- zur Öffentlichen Bibliothek im Venedig des 18. Jahrhunderts. Sie beschäftigt sich mit den Sammlungen und den Lesern und unterstreicht, dass die sogenannte Öffentlichkeit nur durch die Gelehrten und ihre Studien repräsentiert war (S. 113-129). Besonderes Interesse darf der Beitrag von Olga Granasztói (Ungarische Akademie der Wissenschaften/Universität Debrecen) beanspruchen. Sie fokussiert den Zeitabschnitt von 1770 bis 1810, der sich weitgehend mit der sogenannten ungarischen Aufklärung deckt. Sie zieht eine Bilanz der bisherigen Forschungen über die Verbreitung französischer Bücher in den Privatbibliotheken der ungarischen Aristokratie und zeigt die Perspektiven für weitere Forschungen auf. Unter anderem entwirft sie eine Typologie der adeligen Sammler, betrachtet kritisch quantitative Methoden und ihre Grenzen. Sie schließt mit einem Blick auf das weitere Schicksal dieser Bibliotheken im 20. Jahrhundert (S. 181-205). Der von Philippe Dufieux (Präsident der Société historique, archéologique et littéraire de Lyon) als verkannt bezeichnete Architekt Joseph-Jean Pascal Gay (1775-1832) hinterließ eine bedeutende humanistische Bibliothek, deren Auktionskatalog 1833 in Lyon gedruckt wurde. Er verzeichnet mit 958 Einträgen Bücher, Manuskripte und Druckgraphiken. Dufieux analysiert das Verzeichnis und schlüsselt es auch statistisch auf. Damit dokumentiert er die umfassende Bildung des Architekten, der nicht weniger als 31 Ausgaben des Vitruv besaß, darüber hinaus eine beachtliche Zahl von Inkunabeln (S. 207- 
218). Zwei weitere Beiträge sind kirchlichen Bibliotheken gewidmet. Natale Vacalebre (Doktorand der Universität Udine) verfolgt in seinem italienischen Aufsatz noch einmal die Ursprünge der Bibliotheken in den Bildungseinrichtungen der Societas Iesu. Er wertet die „Constitutiones" aus und formuliert Thesen über die Herkunft des ignazianischen Bibliotheksmodells, das normativ für alle Niederlassungen des Ordens sein sollte (S. 51-68). Fabienne Henryot (Kantons- und Universitätsbibliothek Lausanne) untersucht das wenig bekannte Thema des Umgangs kirchlicher Bibliotheken mit Schenkungen und Legaten bis in die Moderne. Dabei geht sie zwei Fragen nach. Die eine ist soziologisch: Wer waren die Donatoren? Die zweite betrifft die Bibliothekspraxis: Wie wurden die Schenkungen in den Bestand integriert und eingearbeitet (S. 69-92)? Die beiden nächsten Beiträge verbindet vom sprachlichen und kulturgeschichtlichen Hintergrund her eine gewisse Verwandtschaft. Maria Luise Cabral (früher Nationalbibliothek Lissabon) verfolgt den Entstehungsprozess der Real Biblioteca Publica da Corte, der Königlichen Öffentlichen Bibliothek des Hofes von Portugal in Lissabon, zwischen 1796 und 1803. In diesen Jahren verwandelte sich die Bibliothek von einer vollständig desorganisierten Institution zu einer für ihre Zeit fortschrittlichen Serviceeinrichtung, parallel zu einem unter dem Einfluss der Aufklärung sich modernisierenden Staatswesen (S. 143-161). Marisa Midori Deaecto (Universität São Paulo) überschreibt ihren Beitrag „La ville et les livres, ou comment former une bibliothèque“ (S. 163-180). Es sind historische Anmerkungen über die Entstehung und über den Katalog der ersten öffentlichen Bibliothek in São Paulo (1825-1887). Der Gründung lagen drei Motive zugrunde: Schaffung der institutionellen Grundlagen der brasilianischen Monarchie, Integration der provinzialen Zentren und ihrer Eliten in den Gesamtstaat sowie die kulturelle Laisierung. Als Letztes soll noch von zwei Beiträgen die Rede sein, die über den bisher abgesteckten Rahmen hinausweisen. Seit jeher bewahrten Bibliotheken auch Kunstgegenstände, archäologische Objekte sowie „naturalia et mirabilia“. Als öffentliche Orte stehen sie heute nicht nur Wissenschaftlern sondern auch Touristen, kulturell Interessierten oder schlicht Neugierigen offen. Andrea de Pasquale (Generaldirektor der Biblioteca Nazionale Centrale Rom) geht diesen Aufgaben der staatlichen Bibliotheken in Italien im 19. und 20. Jahrhundert nach. Nach einem knappen Abriss der historischen Entwicklung listet er in chronologischer Abfolge 18 Bibliotheken mit entsprechenden musealen Aufgaben auf und kommentiert sie. Darunter befinden sich „Highlights“ wie die Laurenziana in Florenz oder die Marciana in Venedig (S. 229-253). Roger Chartier hat die Digitalisierung eine „epistemologische Revolution“ genannt.
Darauf bezieht sich Anne-Marie Bertrand (Universität Lyon/ENSSIB) in ihren „vorläufigen Überlegungen“ zu den Auswirkungen der Datenverarbeitung auf die Beschäftigung mit der Geschichte der Bibliotheken. Im Einzelnen geht sie unter anderem auf die Sammlungen, die Vermittlung, den Zugang zu den Quellen und die Lebensdauer der Materialien ein. Frei nach Descartes kommt sie zu dem Schluss, dass die Digitalisierung nicht nur den methodischen Zweifel provoziert, sondern auch neue Recherchethemen generiert (S. 255-265).

In gebotener Kürze soll noch das zweite Dossier „Études d'histoire du livre" vorgestellt werden, das etwa ein Drittel des ersten umfasst. Rodolfo Savelli (Universität Genua) beschreibt (in Italienisch) die verwickelte Publikationsgeschichte der Werke des savoyischen Strafrechtlers Geoffroy de Bavoz (Godofredus a Bavo) in der ersten Hälfte des 17. Jahrhunderts zwischen Chambéry, Turin und Genf (S. 269-288). Die Bibliothèque bleu war in Frankreich der frühe Versuch, populäre Schriften durch Kolportage zu verbreiten. Geneviève Deblock (Absolventin der EPHE) untersucht als Beispiel einer solchen Schrift „Le bâtiment des recettes“. Diese Schrift war eine beliebte Sammlung technisch-praktischer Hinweise, angeblich geheimer Erkenntnisse, die durch vier Jahrhunderte vom 16. bis zum 19. Jahrhundert verbreitet wurde. Die „Rezepte“ stammten vielfach von Gauklern und Scharlatanen, enthielten aber auch Nützliches und Unterhaltsames (S. 289-313). Alain Riffaud (Université de Maine) schreibt über das wechselvolle Schicksal Jean Ribous (1630-1702), des Buchhändlers, der die Werke Molières verlegt hat (S. 315-363). Ebenfalls ein Buchhändler (Amateurbuchhändler, wie er sich selbst bezeichnete) - und Bibliophiler -, Charles Chardin (1749-1826) ist Gegenstand des Beitrags von Livia Castelli (Bibliothekarin an der Università La Sapienza in Rom). Chardin war der literarische Agent des englischen Schriftstellers und Sammlers William Beckford. Der umtriebige Mann spielte nicht nur eine Rolle im Buchhandel sondern auch als Sammler, in der Bibliotheksgeschichte und während der Französischen Revolution. Letztere brachte ihn sogar vor ein Revolutionstribunal (S. 365-374). Das Dossier beschließt ein Aufsatz von Thierry Dubois (Bibliothèque de Genève) über die Verbreitung nützlicher Kenntnisse im 18. Jahrhundert. Im Mittelpunkt steht der reformierte Pfarrer und Naturwissenschaftler Élie Bertrand (17131797), der in enger Verbindung mit der Ökonomischen Gesellschaft von Yverdon und ihrer Bibliothek stand, und der ihr sein den Naturwissenschaften gewidmetes „Cabinet de curiosités“ vermachte. Dieses sollte sowohl pädagogischen wie ökonomischen Zwecken dienen (S. 375-408).

Der eingangs erwähnte, umfangreiche Einzelbeitrag außerhalb der Dossiers „Un moment dans l'intimité de 
deux grandes dynasties de libraires: les Didot et les Jombert entre Directoire et Premier Empire, à travers quinze lettres inédites" beruht auf einer von Frédéric Barbier an der EPHE betreuten Doktorarbeit von Greta Kaucher. Mit Charles-Antoine Jombert (1712-1784) und François Didot (1685-1757) begann 1746 eine Allianz der beiden Buchhändlerfamilien, die 1779 durch eine Ehe unter den Nachkommen intensiviert wurde. Kauder legt dar, wie sich die Verbindungen entwickelt haben und ediert im Anschluss 15 bisher nicht veröffentlichte Briefe, die von 1786 bis 1913 gewechselt wurden. Sie geben Einblick nicht nur in die familiären Beziehungen und in das Familienleben, sondern, wenn auch in geringerem Umfang, in die Geschichte des Verlags und der Druckerei. Darüber hinaus sind sie als lebendige Zeitdokumente wertvoll (S. 411-457).

Der letzte Abschnitt des Sammelbands bringt sieben sehr ausführliche Rezensionen einschlägiger Neuerscheinungen. Als Resümee ist festzuhalten, dass die Beiträge ein breites Spektrum von Themen abdecken. Mögen sie inhaltlich da und dort von eher lokalem oder regionalem Interesse sein, so zeigen sie doch vielversprechende methodische Ansätze für eine „modernisierte“ Bibliothekshistoriographie. Zurecht also stellt Frédéric Barbier als Herausgeber fest: „Depuis plusieurs années, les questions posées par les historiens aux bibliothèques se sont donc profondément renouvelées“(S. 10).

Als kleine kritische Anmerkung: Anstelle der kargen Angaben zu den Autoren zu Beginn jedes Beitrags hätte man sich etwas ausführlichere Curricula vitae am Ende des Bands gewünscht.

\section{Joachim Eberhardt und Detlev Hellfaier (Hrsg.):}

1614-2014. 400 Jahre Lippische Landesbibliothek. (Auswahl- und Ausstellungskataloge der Lippischen Landesbibliothek Detmold; Bd. 38). [Detmold:] Lippische Landesbibliothek, 2014. 188 S., zahlr. Ill. ISBN: 978-3-9806297-6-8; ISSN: 1435-7615

Besprochen von Dr. Werner Arnold: Am Kälberanger 4 b, D-38302 Wolfenbüttel, E-Mail: guelferbytanus@gmx.de

Die Fürstenbibliotheken der deutschen Territorien waren die Wissensspeicher und intellektuellen Zentren der Frühen Neuzeit, viel leistungsfähiger als die Universitätsbibliotheken und durch ihre Sammlungen von überregionaler Bedeutung für den allgemeinen Diskurs. Das hier vorzustellende Buch von Joachim Eberhardt und Detlev Hellfaier - Herausgeber und Autoren zugleich - über die vierhundertjährige Geschichte der Lippischen Landesbibliothek Detmold lässt alle charakteristischen Entwick- lungsmerkmale der fürstlichen Sammlungen, die sich zu Landesbibliotheken entwickelten, erkennen, oder besser gesagt, Eberhardt und Hellfaier haben diese Charakteristika präzise herausgearbeitet und formuliert.

Das Buch ist chronologisch gegliedert und integriert die wichtigsten Sachgebiete in die Zeitebenen. Den Anfang bildete die Stiftung der Bibliothek Graf Simons VI. zur Lippe (†1613) durch seinen Sohn 1614 als Bildungsinstitution, was durch die Aufstellung der Bücher in den Räumen der Lateinschule noch hervorgehoben wurde. Ein erster Katalog von 1597 verzeichnet 493 Titel, während 1613 bereits 2200 Bände vorhanden waren, also in kurzer Zeit ein prozentual hoher Zuwachs erreicht war. Die Autoren bewerten diese Zahl als „nicht viel“; das kann aber nur aus heutiger Sicht gelten, während der Umfang für die Zeit unmittelbar vor Ausbruch des Dreißigjährigen Kriegs beachtlich ist und die klare Tendenz zu einer Universalbibliothek erkennen lässt. Die stark vertretenen Disziplinen Theologie, Jurisprudenz und Geschichte zeigen das für die Frühe Neuzeit traditionelle Wissenschaftsverständnis, aus dem sich im späten 17. und im 18. Jahrhundert ein differenziertes Fächerspektrum entwickelte. Auch der intellektuelle Einfluss aus dem Umfeld der Gelehrten auf den Bestandsaufbau (reformierte Theologie) sowie der Ankauf von Privatbibliotheken bilden spezifische Merkmale für die Entwicklung der Fürstensammlungen. Wenn man ihre Finanzsituation beurteilt, fällt (überall) die mangelnde Basisfinanzierung auf, da monetäre Entscheidungen subjektiv vom Landesherrn ohne definierte Planungen gefällt wurden, es also (in Detmold bis 1824) bis zur Einrichtung einer vorausplanenden und verpflichtenden Kassenführung keinen ausreichenden ordentlichen Haushalt gab. Andererseits wurden häufig außerordentliche Mittel zur Verfügung gestellt, so dass der Bestandszuwachs insgesamt gewährleistet war.

Die Autoren beschreiben die Sondersammlungen (Handschriften, Inkunabeln, Quellen zu lippischen Geschichte), also die Pfunde, mit denen die Landesbibliotheken heute wuchern können und verweisen auf Mäzene, die diese Quellen zugänglich gemacht haben. Das Prinzip der Stärkung der Institution durch besondere Sammlungen, die Alleinstellungsmerkmale bedeuten, wurde konsequent beibehalten. Ferdinand Freiligrath schenkte Handschriften früher Werke als Dank für die Anregungen, die er durch die Bibliothek erhalten hatte und legte damit indirekt die Grundlage der Dokumentensammlung des Lippischen Literaturarchivs; zu den überregional bekannten Sonderbeständen gehören auch die Grabbe-Sammlung sowie das Lortzing-Archiv, die Kooperationsmöglichkeiten mit verwandten Einrichtungen ermöglichen. In Detmold hat man viel Mühe in die Erschließung dieser Sammlungen 
investiert, und die beigefügte Bibliographie enthält die entsprechenden Veröffentlichungen. Interessante Einzelaspekte sind beispielsweise der Nutzen, den die Bibliothek aus den Pflichtexemplaren zog, die die starke Meyer'sche Buchhandlung in Lemgo nach Detmold abliefern musste, sowie wichtige Einzelquellen, wie das 1867 geschenkte Stammbuch Engelbert Kaempfers (1651-1716) aus Lemgo, der als berühmter Forschungsreisender und Autor einer Geschichte Japans erst in den vergangenen zwei Jahrzehnten von der Forschung wirksam wiederentdeckt wurde.

Alle Fürstenbibliotheken sind durch die Aufklärung geprägt worden. In Detmold geht dieser Einfluss vor allem auf die Fürstin Pauline (1769-1820) zurück, die für ihren Sohn Leopold die Regentschaft führte. Sie veranlasste im Rahmen eines neuen Bildungskonzepts die Zusammenführung der Detmolder Bibliotheken und ließ sie der Öffentlichkeit zur Verbesserung der Bildungsmöglichkeiten zugänglich machen. Das entscheidende Verdienst zur Realisierung der Zukunftsentwicklung gebührt Otto Preuß, der die Bibliothek 1838 bis 1890 zwar nur im Nebenamt, aber mit großem Engagement leitete und durch Erschließung der Quellen sowie deren Dokumentation die Basis für den Aufbruch in die Moderne schuf; Preuß war hauptamtlich Vorsitzender des Hofgerichts und der Justizkanzlei. Das Zeugnis aufklärerischen Denkens, das den Anstoß für die künftige Entwicklung gab, stellt die Privatbibliothek (ca. 1600 Bände) Paulines dar, die systematisch die Neuerwerbungen ihrer Zeit kaufte, die Bücher las und über sie diskutierte, deren Nutzen also durch Argumente und Gegenargumente prüfte und prüfen ließ.

Diese Orientierung der Bibliothek als Ort des Lernens und der wissenschaftlichen Praxis zeichnet die Entwicklungslinien bis zur Gegenwart vor. Nach dem Ersten Weltkrieg wurde die Fürstliche Bibliothek zur Landesbibliothek und übernahm umfangreiche Bildungsaufgaben für die Bevölkerung. Die demokratische Entwicklung wurde während der NS-Zeit unterbrochen - in Lippe-Detmold konnte die NSDAP bereits bei Landtagswahlen im Januar 1933, noch vor der Ernennung Hitlers zum Reichskanzler, knapp $40 \%$ der Stimmen gewinnen - als der Bibliotheksdirektor Eduard Wiegand die Institution zur Indoktrinierung einsetzen wollte und dazu u.a. eine Sammlung zur NS-Geschichte aufbaute, die heute selbst zum historischen Dokument geworden ist.

Seit geraumer Zeit versucht die Lippische Landesbibliothek, ihre Handlungsmöglichkeiten durch Synergien zu stärken. Dazu gehören die Kooperation mit der Bibliothek der Hochschule für Musik und die Integration der Bibliothek der Lippischen Landeskirche. Eine bemerkenswerte Entwicklung konnte für das Gebäude der Bibliothek, das „Prinzenpalais“ in der Hornschen Straße, in dem sie seit 1886 ihren Platz hat, erreicht werden, denn durch die Magazinbauten vor allem in den vergangenen zehn Jahren wurden Stellflächen für ca. eine Million Einheiten geschaffen. Mit diesen Möglichkeiten wird die Lippische Landesbibliothek weiter wachsen.

Tiziana Stagi: Una battaglia della cultura. Emanuele Casamassima e le biblioteche. Roma: Associazione italiana biblioteche, 2013.607 S. Mit Anlagenteil, s.w. Abb. u. Personen- und Institutionenverzeichnis.

ISBN 978-88-7812-222-2

Besprochen von Klaus Kempf: Bayerische Staatsbibliothek, Ludwigstr.16, D-80539 München, E-Mail: Klaus.Kempf@bsbmuenchen.de

Italiens Bibliotheken existieren heute für deutsche Bibliothekare gemeinhin am Rande des beruflichen Interessenshorizontes. Noch weniger kennt man Namen und Rang italienischer Bibliothekare. Sieht man einmal von Antonio Panizzi ab, der allerdings eher als Vertreter des britischen Bibliothekswesens gelten muss, so sind selbst international renommierte Vertreter des Berufsstandes aus Italien, wie Guido Biagi, der wie kein zweiter schon zu Beginn des 20. Jahrhunderts die internationale Kooperation als eine wesentliche Komponente bibliothekarischen Arbeitens erkannt und propagiert hatte oder Renato Serra, der vormalige Direktor der Biblioteca Malatestiana in Cesena und einer der bedeutendsten italienischen Intellektuellen seiner Zeit oder ein Schriftsteller, wie Federico De Roberto, dessen Rom an „Die Vizekönige“ zum erweiterten Kanon der Weltliteratur gehört und der im bürgerlichen Leben Bibliothekar an der wissenschaftlichen Stadtbibliothek von Catania war, in hiesigen Breiten so gut wie unbekannt. Dies war nicht immer so. Vor dem Ersten Weltkrieg, in den 90er-Jahren des 19. Jahrhunderts, also zu Zeiten des großen Aufbruchs im deutschen Bibliothekswesen fuhr einer seiner namhaftesten Vertreter im staatlich-preußischen Auftrag zu Fortbildungszwecken nach Italien. Die Rede ist von Carl Dziatzko. Letzterer war vom 1. März bis 15. Mai 1892 gut 10 Wochen im jungen Königreich Italien unterwegs, um sich dort vor Ort einen Eindruck von moderner (!) Bibliotheksarbeit zu verschaffen. ${ }^{1}$ In späterer Zeit kam eine so systematische Inaugenscheinnahme dessen, was bibliothekarisch südlich des Brenners passiert, lange nicht

1 Vgl. Karl Dziatzko: Eine Reise durch die größeren Bibliotheken Italiens. Sonderabdruck aus Sammlung bibliothekswissenschaftlichen Arbeiten. Heft 6. 
mehr vor. Das galt selbst für die Zeit des Faschismus bzw. des Nationalsozialismus als man sich zumindest bilateral bibliotheksfachlich erstaunlich wenig zu sagen hatte. Begegnungsfeld für die Vertreter der beiden Bibliothekswelten vor und nach dem Zweiten Weltkrieg blieb primär die internationale Bühne, wie z.B. die Jahreskongresse der IFLA, die im Übrigen 1929 in Italien mit wohlwollender Förderung des Duce und dem - wörtlich zu nehmenden Segen des damaligen Papstes, Pius XII., offiziell das Licht der Welt erblickten.

Dies änderte sich schlagartig mit einer in Friedenszeiten fast einmaligen Katastrophe. Das italienische Bibliothekswesen und einer seiner maßgeblichen Vertreter im zwanzigsten Jahrhundert waren mit der im November 1966 über die Welthauptstadt der Renaissance, Florenz und die dort gelegene Nationalbibliothek urplötzlich hereingebrochene Flutkatastrophe von einem auf den anderen Tag in Deutschland, ja weltweit in aller Munde. Die fast unmittelbar am Ufer des Arno gelegene Biblioteca Nazionale Centrale di Firenze (BNCF) war vollständig überschwemmt und ihre Räume, vor allem die in den Kellerräumen gelegenen Magazine, von einer Schlammflut von bis dahin nicht gekanntem Ausmaß heimgesucht worden. Es drohte der Totalverlust von einmaligem Sammelgut. Ein nicht unerheblicher Teil des schriftlichen Weltkulturerbes war in Gefahr, für immer verloren zu gehen. In dieser äußerst dramatischen Situation und unter teilweise chaotischen Rahmenbedingungen behielt ein Mann die Nerven und den Überblick, der Bibliotheksleiter Emanuele Casamassima. Er, seine Familie, seine Mitarbeiter und die später „Schlammengel“ - angeli del fango - genannten, Hunderte von Freiwilligen aus aller Welt brachten es unter widrigsten Umständen fertig, dass ein Großteil der buchstäblich in Schlamm und Wasser versunkenen und eigentlich verloren geglaubten Bestände doch noch gerettet wurden und die BNCF mit dem bei ihr mit internationaler Unterstützung eingerichteten Zentrum für Buchrestaurierung mit seinen innovativen Techniken zur Massenreinigung und -konservierung letztlich zum Wegbereiter der modernen Bestandserhaltung wurde.

In einer bereits im Jahr 2013 von der italienischen Bibliotheksvereinigung (AIB) verlegten, sehr sorgfältig unter Nutzung neuer Quellen und Auswertung aller nur denkbaren Archivalien erarbeiteten, umfänglichen Monographie wird ausführlich das Werden und Wirken von Emanuele Casamassima in all seinen Facetten, jedoch mit eindeutigem Schwerpunkt auf der erwähnten Flutkatastrophe vorgestellt. Über die detaillierte Schilderung des eigentlichen Rettungsvorgangs und die entscheidende Rolle, die Casamassima dabei spielte, hinaus wird überzeugend herausgearbeitet, dass Casamassima das Jahrhundert- desaster, das seine Bibliothek heimgesucht hatte, dazu nutzen wollte, die BNCF neu zu positionieren und sie mit einem zeit- und technikgemäßen Organisations- und Personalkonzept zu versehen. Er schaute bei der Suche nach Vorbildern dabei nicht nur in die ihm affine angelsächsische Bibliothekswelt, sondern reiste im Auftrag des zuständigen Ministeriums mehrfach auch in die Bundesrepublik Deutschland, um sich vor Ort z. B. in Bochum einen unmittelbaren Eindruck von den ersten EDV-Anwendungen im deutschen Bibliothekswesen zu verschaffen. Die wichtigsten diesbezüglichen Erfahrungsberichte und daraus hervorgegangenen Denkschriften Casamassimas sowie weitere Texte und Materialien von seiner und von dritter Hand sind dem Band in einem fast zweihundertseitigem Anlagenteil beigegeben.

Dort werden allerdings auch das Scheitern seiner Überlegungen und Pläne für eine tiefgreifende Reorganisation der BNCF und letztlich Neuordnung des staatlichen Bibliothekswesens dokumentiert. Die Widerstände resultierten vor allem aus einer eisern an ihrem zentralistischen, streng hierarchischen Organisationskonzept festhaltenden römischen Ministerialbürokratie, die bis in unsere Tage maßgeblich für den Zustand des italienischen Bibliothekswesens verantwortlich zeichnet. Emanuele Casamassima wird als eine charismatische, mit vielen Talenten versehene Persönlichkeit vorgestellt, die ihre über den Tag hinausreichenden bibliothekspolitischen und -fachlichen Konzepte im internationalen Kontext bzw. unter Bezugnahme auf die aktuelle oder sich andeutende Entwicklung in anderen Ländern, vor allem dem angloamerikanischen Raum entwickelte, damit jedoch im heimischen beruflichen und übergeordneten politischadministrativen Umfeld nur bedingt auf Verständnis stößt und am Ende damit nicht durchdringt. Casamassima erkennt sein Scheitern und verabschiedet sich konsequent von seinen Ämtern im Bibliothekswesen. Er kehrt zu seinen eigentlichen Studienfächern zurück und verbringt seine letzten Berufsjahre als Universitätsprofessor für Paläographie in Triest und Florenz.

Der Band wird komplettiert durch einen einführenden Beitrag von Mauro Guerrini, eine umfassende Bibliographie, einen Bild- bzw. Fototeil mit bisher weitgehend unveröffentlichten Fotos aus privater Hand sowie ein erschöpfendes Personen- und Institutionenregister, das nochmals die weitreichende berufliche, bibliotheksfachliche Vernetzung von Casamassima eindrucksvoll aufzeigt. Mit der Arbeit von Stagi liegt ein Werk vor, das so ausführlich und so farbig wie bisher kein zweites diese überaus wichtige und letztlich weichenstellende Zeitspanne im italienischen wissenschaftlichen Bibliothekswesen, nämlich die sog. Jahre des Aufbruchs, d.h. das knappe Jahrzehnt 
von 1965 bis zum ersten Ölpreisschock, schildert. Dem hier besprochenen Werk haftet eigentlich nur ein Mangel an, wenn man dies so nennen darf - es ist auf Italienisch verfasst und von daher keine einfache Lektüre. Es sollte gleichwohl in keiner (wissenschaftlichen) Bibliothek fehlen, der die Dokumentation und die Vermittlung des Lebens und des Wirkens herausragender Persönlichkeiten unseres Berufsstandes - und Emanuele Casamassima gehört hier ohne Zweifel dazu - noch am Herzen liegt.

Die Manuscripta Magdeburgica der Staatsbibliothek zu Berlin - Preußischer Kulturbesitz. Teil 4 (Ergänzungsband). Die Codices electorales Magdeburgenses. Nach den Beschreibungen von Valentin Rose neu bearb. von Ursula Winter. Wiesbaden: Harrassowitz, 2012.171 S. (Staatsbibliothek zu Berlin - Preußischer Kulturbesitz, Kataloge der Handschriftenabteilung, Reihe 1. Handschriften, Band 4, Teil 4 = Ergänzungsband). ISSN 0172-1119; ISBN 978-3-447-06731-7

Besprochen von Doz. Dr. Konrad Marwinski: Engelbert-Schoner-Weg 12, D-99425 Weimar

Nachdem Brandenburg im Westfälischen Frieden von 1648, der den Dreißigjährigen Krieg beendete, Hinterpommern, die säkularisierten Bistümer Halberstadt, Minden und Kammin hinzugewonnen und die - 1680 erst eingelöste - Anwartschaft auf das Erzbistum Magdeburg mit Halle zugesichert bekommen hatte, wurden die Verwaltung und die Strukturen des Kurfürstentums reorganisiert. Die „Churfürstliche Bibliothek zu Cölln an der Spree“, 1701 in Königliche Bibliothek umbenannt, entstand 1659/1661 aus der Hofbibliothek des brandenburgischen Kurfürsten Friedrich Wilhelm (1620-1688, Kurfürst ab 1640), der den Bestandsaufbau forcierte, indem er die zuständigen kirchlichen und städtischen Beamten der hinzugewonnenen Territorien anwies, die in ihren Verantwortungsbereichen relevanten Objekte zu erfassen und diese, falls sie geeignet erschienen, zur Verfügung zu stellen. Die Folge davon war, dass im Rahmen dieser Aktion aus dem Erzbistum Magdeburg zahlreiche Handschriften in die Kurfürstliche Bibliothek gelangten.

Der Handschriftenkatalog der Manuscripta Magdeburgica der Staatsbibliothek zu Berlin - Preußischer Kulturbesitz erschien zwischen 2001 und 2008 in 3 Teilen. ${ }^{1}$ Die außerordentlich genauen Beschreibungen verfasste die

1 Vgl. meine Rezension in: BIBLIOTHEK - Forschung und Praxis 36 (2) (2012) S. 257-258. frühere Wissenschaftliche Mitarbeiterin der Berliner Handschriftenabteilung Dr. Ursula Winter, Teil 1 allein, und Teil 2 und 3 gemeinsam mit Kurt Heydeck. Die Bearbeiterin hatte mit der Katalogisierung schon Mitte der 1980er-Jahre begonnen und später unter dessen Mitarbeit fortgesetzt. Die meisten der Beschreibungen von Ursula Winter waren jedoch erst nach ihrem Ausscheiden aus dem Berufsleben in ehrenamtlicher Tätigkeit entstanden. In den Einleitungen zu den drei Teilen der Beschreibungen der in der Mehrzahl aus dem 14. und 15. Jahrhundert stammenden Manuscripta Magdeburgica wird ein komprimierter Überblick über die komplizierte Bestandsgeschichte der Sammlung gegeben, die bereits im 10. Jahrhundert mit dem Domstift Magdeburg, das aus dem Benediktinerkloster St. Mauritius entstand, ihren Anfang nahm. ${ }^{2}$

Ursprünglich war der Druck eines vierten Teiles des Kataloges der Manuscripta Magdeburgica wohl nicht vorgesehen. Bei den Arbeiten an dem zunächst nur dreiteiligen Katalog der Manuscripta hatte man jedoch im Vergleich mit dem „Verzeichnis der lateinischen Handschriften der königlichen Bibliothek zu Berlin (Bd. 2,1-3, Berlin 1901-1905)“ von Valentin Rose (1829-1916) wertvolle neue Erkenntnisse gewinnen können, die in dem vorliegenden Band, der seinem Gedenken gewidmet ist, ihren Niederschlag fanden.

Er enthält die Beschreibungen von 47 lateinischen theologischen Handschriften aus dem 10. bis 16. Jahrhundert (mit Schwerpunkt im 13. bis 15. Jahrhundert), die zum größten Teil nach 1685 in die Bibliothek kamen. Es handelt sich hierbei zumeist um Pergamentcodices (35), dazu noch aus der Einbandmakulatur 49 Fragmente (39 Pergamentund 10 Papierfragmente).

In seiner Struktur entspricht der vierte Teil der Manuscripta Magdeburgica den drei vorangegangen Teilen. Auch hier wendet Ursula Winter die bewährte Methode der Tiefenerschließung an. Die Beschreibungen sind typographisch übersichtlich gestaltet. Der Einleitung folgen das Abkürzungsverzeichnis (S. 14-16), das Verzeichnis der abgekürzt zitierten Literatur (S. 17-24), das Signaturenverzeichnis der beschriebenen Handschriften (S. 25-26; mit Angabe der Rose-Nr.), Beschreibungen der Handschriften (S. 27-136) in der Abfolge des Signaturenverzeichnisses, und zur Erschließung des Materials ein Initienregister (S. 138-151) sowie ein Personen-, Orts- und Sachregister

2 Zur Geschichte der Sammlung vgl. auch Winter, Ursula: Die Handschriften des ehemaligen Domgymnasiums Magdeburg in der Staatsbibliothek zu Berlin - Preußischer Kulturbesitz. Geschichte und erste Ergebnisse der Katalogisierung. In: Mittellateinisches Jahrbuch 28 (1993) S. 103-112. 
(S. 152-169). Den Abschluss bildet eine Übersicht der Signaturen-Konkordanzen (S. 170-171).

$\mathrm{Zu}$ den ältesten Stücken im Bestand zählt ein Evangeliarium (Codex Wittekindeus) aus dem letzten Drittel des 10. Jahrhunderts (Ms. Theol. Lat. fol. 1). Der Pergamentband ist mit Kanontafeln, Evangelistenbildern, Flechtwerk- und Rankeninitialen ausgestattet. „Die zum ursprünglichen Prachteinband gehörenden vier Elfenbeinplatten sind in den Vorderdeckel eingelassen“, sie gehören zur „Gruppe der Magdeburger Elfenbeintafeln“.

Als Autoren theologischer Texte begegnen mehrfach Anselm von Canterbury (1033-1109), Thomas de Aquino (1225/57-1274) und Jean Charlier de Gerson (1363-1429). Aus dem Besitz von Thomas Hirschhorn (gest. vor 1493), ab 1441 Leibarzt des Magdeburger Erzbischofs Günther XXXIII. von Schwarzburg (1382-1445) und des Domkapitels stammt eine zusammengesetzte fünfteilige Handschrift aus der Mitte des 15. Jahrhunderts (Ms. Theol. Lat. fol. 141; $443 \mathrm{Bl}$.), die 56 Einträge erhalten hat.

Profane Literatur fehlt ganz, es existiert lediglich ein Fragment (Ms. Lat. oct. 46; 10 Pergamentblätter) aus der
„Pharsalia“ des römischen Dichters Lucanus (39-65 n. Chr.), einer Handschrift aus dem letzten Drittel des 13. Jahrhundert. Bemerkenswert ist noch eine Ergänzung zu Teil 1. Bei Katalogisierungsarbeiten in der Russischen Nationalbibliothek in Moskau wurde eine juristische Sammelhandschrift (38 Nummern, 2. Viertel 15. Jahrhundert) aus dem Magdeburger Domgymnasium gefunden, die bei der Rückgabeaktion 1957/58 wahrscheinlich übersehen worden war (Sign.: Ms. Magdeb. 43, Moskau). ${ }^{3}$

Mit dem vierten Teil der Manuscripta Magdeburgica haben Ursula Winter, die Staatsbibliothek zu Berlin Preußischer Kulturbesitz und der Harrassowitz Verlag Wiesbaden ein Werk in ansprechendem Layout zum Abschluss gebracht, das für weiterführende historische Forschungen ein bis dahin relativ unbekanntes Quellenmaterial in vorbildlicher Weise erschließt.

3 Die Rückführung von Handschriften aus der UdSSR vgl. Schipke, R., u. U. Winter, Ursula: Verlagert, verschollen, zurückgekehrt. Handschriftenbestände der Berliner Staatsbibliothek. In: Jahrbuch Preußischer Kulturbesitz 45 (2008/2009) S. 331-333. 